www. revis ta d y o. com

\title{
Flexibilidad Funcional y Flexibilidad Financiera en las Empresas Media- nas y Grandes del Sector Metal en Cataluña
}

Functional flexibility and financial flexibility in large and medium-sized enterprises of the metal sector in CataIonia

Francisco Llorente Galera

AQR Research Group- IREA, Departamento Econometría Estadística y Economía Española, Facultad de Economía y Empresa, Universidad de Barcelona

fllorente@ub.edu

Fecha de recepción: 15-10-2015

Fecha de aceptación: 22-02-2016

Resumen: El artículo analiza en las empresas medianas y grandes del sector metal de Cataluña la intensidad de implantación individual y conjunta de diversas modalidades de la flexibilidad funcional y flexibilidad salarial, sus posibles relaciones, y establecer una tipología de empresas. Además, conocer si hay diferencias significativas en los ritmos de trabajo, nivel de estrés, absentismo, productividad laboral, margen de explotación y Gastos de personal/Ingresos de explotación, según el grado de aplicación de los ítems de flexibilidad seleccionados. La implantación conjunta de la mayoría de ítems de flexibilidad laboral considerados es muy reducida, obteniéndose una hibridación de empresas por su incorporación.

Palabras clave: Metal, Cataluña, Flexibilidad, Trabajo

\begin{abstract}
This paper summarizes the theoretical characteristics of the types functional flexibility and wage flexibility. In the empirical part we check for a sample of companies in the Catalonia metal sector with at least 100 employees, if several modalities about functional flexibility are implemented. Mainly the job rotation, autonomy over work, and delegate indirect tasks to the operator. In addition also the modalities of variable pay for the operator. The individual and joint application of the items is analyzed, making a descriptive statistical analysis and bivariate association. In most companies operators are intensively responsibles for the order and clean their workplace, as well as self control, followed by prepares the machine, to search the origin of defects and their solution. Almost half firms haven't incorporated teamwork, while cross functional teams and improvements teams are more incorporated, although the proportion that applies a high degree is low. The operator does not usually engage in maintenance of machinery nor collaborate in the development and improvement of the standard. There are limited autonomy in work organization and planification, fixing work methods and rhythm of work. The wage flexibility is low, although the pay for performance/productivity is the modality most used. The pairs of modalities of wages that are significantly are the incentives: "to fulfill team goals" and "quality", "profit sharing" and "knowledge / skills /abilities ". Moreover it is tested whether there are significant differences according to central tendency of various items (rhythm of work, stress level, absenteeism level as well as the ratios: percentage of variable pay, labor productivity, ROS, and personnel costs/revenues) segmented according to the modality functional flexibility or wage flexibility considered. The main conclusions are that rhythms of work and absenteeism are lower when job rotation is reduced, there aren't significant differences in productivity, but there are differences in central tendency measures of the profit margin when segmenting by the items: autonomy in work organization and planification, rhythm of work, self control, order and clean, and operator maintenance of machinery. The value of this ratio is lower when such items are reduced.
\end{abstract}

Few companies incorporated most labor practices intensely. Therefore, most firms require greater joint incorporation of labor flexibility dimensions to achieve complementarities and synergies. Homals analysis is used to establish relationships between more than two qualitative variables and reduce the dimensionality by incorporating measures of discrimination. The first dimension includes mainly: the operator is responsible of seeks the origin of defects, to solve the defects found, to program the machine, the basic maintenance of the machine and analyzed data. The second dimension includes: teamwork in the production area, teams improvement voluntary, the operator involvement in continous improvement and cross teams to solve problems. We identify a typology of firms, using cluster analysis with the results of Homals. No significant differences were obtained when segmented by the 10 groups offered by the cluster. 


\section{Introducción}

Las empresas están inmersas en un entorno con alta incertidumbre e intensa competencia empresarial (D'Aveni, 1994), donde cambian las demandas de los consumidores, necesitándose ofrecer productos con adecuada calidad, precio, servicio y variedad. La flexibilidad es un factor crítico para una estrategia de producción exitosa (Karuppan, 2004) y la flexibilidad laboral un elemento clave de la misma (Goudswaard et al., 2009), cuyo objetivo es responder a los cambios de las condiciones de la demanda, competencia y tecnológicos (Asplund y Oksanen, 2003).

Las empresas requieren ofrecer productos con suficiente valor añadido, adaptarse ágilmente a los cambios del mercado, tener unos costes laborales unitarios aceptables y mejorar la productividad, cuestiones que facilita la flexibilidad laboral. Debe destacarse que las matrices de los grupos multinacionales frecuentemente exigen a sus filiales en Cataluña mayor flexibilidad laboral y reducción de costes para asignarles nuevos productos e inversiones.

Atkinson (1984) consideran tres grandes tipos de flexibilidad laboral: numérica, funcional y financiera. Para Voudouris (2007) frente a la estrategia de adoptar la reducción de costes, implantando la flexibilidad numérica, alternativamente las empresas pueden establecer la estrategia de mejorar el perfomance de la organización del trabajo, ampliando las habilidades y competencias de sus trabajadores, para que efectúen una variedad de tareas y participen en la toma de decisiones. Para ello debe incorporarse la polivalencia, el trabajo en equipo y delegar responsabilidades en el trabajador, que forman parte de la flexibilidad funcional, y junto a la flexibilidad financiera ayudan a la competitividad empresarial.

En este artículo el principal objetivo es comprobar en las empresas con al menos 100 trabajadores del sector metal en Cataluña, en que grado se han implantado las modalidades de la flexibilidades funcional y la flexibilidad financiera en los operarios, la asociación bivariante y multivariante entre las mismas, Se desea establecer una tipología de empresas a partir de las prácticas laborales consideradas que más se relacionan entre sí. Se verifica si hay diferencias significativas en los ritmos de trabajo, nivel de stress, absentismo, productividad laboral, margen de explotación y peso de los gastos de personal en los ingresos de explotación, según la implantación de tales modalidades de flexibilidad y entre los grupos obtenidos en el cluster.

El resto del artículo se estructura de la siguiente forma.
En la segunda sección se revisa la literatura sobre la flexibilidad funcional y la flexibilidad salarial. Posteriormente se expone la metodología del análisis empírico, se presentan los resultados del análisis y finalmente se presentan las conclusiones, discusiones y limitaciones del estudio.

\section{Revisión Teórica}

El rígido modelo taylorista-fordista, basado en un sistema jerárquico de fuerte control al operario (Coriat, 1992), desde los años sesenta se ha ido sustituyendo por otros modelos como la especialización flexible (Piore y Sabel, 1984), la producción reflexiva y la producción ajustada (Womack et al., 1990; Boyer y Freyssenet, 2000), caracterizados por incorporar una amplia flexibilidad funcional. La producción reflexiva y la producción ajustada también aplican el salario variable, el cual que junto a la flexibilidad funcional forman parte de las prácticas de recursos humanos denominadas de alto rendimiento (Osterman, 1994, Pfeffer, 1995, Appelbaum et al., 2000).

A continuación se comentan los principales rasgos de los dos tipos de flexibilidad laboral objeto del presente artículo: la flexibilidad funcional y la flexibilidad financiera.

\subsection{Flexibilidad funcional}

La flexibilidad funcional para Fina (2001) es la capacidad que tiene la empresa para asignar a los trabajadores en unas $u$ otras tareas o puestos de trabajo dentro de la empresa. Tsipouri et al. (2005) y Arvanitis (2005) la amplían, opinando que es el proceso por el cual los puestos de trabajo se ajustan a los cambios de la demanda, mediante una reorganización interna de los puestos de trabajo, la polivalencia, el trabajo en equipo y la involucración de los trabajadores en diseñar el puesto de trabajo, la innovación, la tecnología y la organización del trabajo.

La flexibilidad funcional exige personal multifuncional (Singh y Chauhan, 2013) para desempeñar una diversidad de puestos de trabajo. Por ello, se requiere un personal cualificado, necesitándose ofrecerle mayor formación y entrenamiento (Delaney y Huselid, 1996, Michie y Sheeban, 2001, Gong y Hu, 2008).

La flexibilidad laboral depende de factores individuales (antigüedad en la empresa y personalidad del personal), factores organizativos que recogen el énfasis en la calidad, flexibilidad, coste y tiempo, y además factores del trabajo, como la responsabilidad conjunta de los 
trabajadores, la automatización y la complejidad de las tareas (Karuppan, op. cit.).

La flexibilidad funcional permite disponer de operarios con más autonomía y nuevas responsabilidades (Valverde et al., 2000), utilizar mejor sus conocimientos (Frenkel y Kurinvilla, 2002), mejorar la calidad del trabajo (Beardwell y Holden, 2001), incrementar la involucración, motivación (Cosgel y Micelli, 1999) y satisfacción del personal (Bauer, 2004). En cambio, para ciertos autores, genera inconvenientes, como la intensificación del trabajo (Cruz y Pil, 2011).

A continuación se comentan las modalidades relevantes de la flexibilidad funcional.

\subsubsection{La Rotación entre puestos}

La rotación entre puestos supone el reemplazamiento planificado de trabajadores entre diversos puestos de trabajo en un período de tiempo (Bennett, 2003), asignando a cada trabajador diversas tareas entre las que rota con cierta frecuencia (Cosgel y Miceli, 1998).

La rotación entre puestos permite adaptarse mejor a un entorno más incierto (Cosgel y Miceli, 1998), desarrollar multihabilidades en el operario (Koike, 1991), incrementar sus conocimientos y habilidades (Kaymaz, 2010), la capacidad de aprendizaje en la empresa (Jerez Gómez et al., 2004), desarrollar el capital humano (Bennet, op. cit. Hsu et al., 2007) y ajustarse mejor a las necesidades productivas (Eriksson y Ortega, 2006). Posibilita incrementar la productividad (Miller et al., 1973, Bennett, op. cit., Origo y Pagani, 2008), extraer información sobre la productividad de las tareas de los trabajadores (Arya y Mittendorf, 2004), reducir la monotonía en el trabajo (Eriksson y Ortega, 2006) y los problemas músculo esqueléticos (Rissen et al., 2002), aumentar la motivación (Kaymaz, op, cit.) y la satisfacción laboral (Cosgel y Miceli, 1999).

Entre los mayores inconvenientes de la rotación entre puestos está la menor holgura del operario en su trabajo, que le genera estrés (Ortiz, 1999), al intensificarse el trabajo.

\subsubsection{El Trabajo en equipo}

El trabajo en equipo ${ }^{1}$ es un elemento central de las nuevas formas de organización laboral (Cully et al.,
1999). En un entorno laboral que incorpora tecnología más compleja, los operarios deberían estar integrados en equipos, entre cuyas responsabilidades han de estar el control de calidad, el mantenimiento básico, y ciertas tareas de reparación (Mueller, 1992).

El trabajo en equipo, estableciendo equipos en la línea de producción, constituye una forma de organización del trabajo que debe diferenciarse de los equipos interfuncionales para solucionar problemas, que están formados por personas de diferentes áreas y departamentos, que ofrecen conocimientos complementarios, que rompen las barreras que se crean entre los mismos, y son de carácter interjerárquico, que funcionan durante un período temporal limitado. Se implantan para poder solucionar mejor determinados problemas complejos que surgen, permitiendo identificar más satisfactoriamente las causas de los problemas y evaluar mejor las soluciones alternativas que ofrecen (Institute of Management Accounts, 1994). Sus miembros adoptan comportamientos colaborativos e integran mejor sus habilidades (Jassawalla et al., 1989), permitiendo una mayor creatividad, rapidez de respuesta y asumen menores costes, especialmente en el desarrollo de nuevos proyectos (Parker, 2003). Incorporan los equipos de proyecto y task force.

Los equipos de mejora incorporan personal cuyo objetivo que ofrezcan ideas para mejorar los procesos $u$ otros ámbitos (Prado, 2000) y son clave para implantar un sistema de mejora continua (Puig Bernabeu et al., 2010).

El trabajo en equipo es un mecanismo de coordinación, cuya estructuración debe permitir tomar decisiones más eficaces (Marschak y Radner, 1972) y ofrecer un output total superior a la suma individual de cada trabajador (Alchian y Demsetz, 1972), al darse sinergias entre los miembros. Posibilita generar mayores conocimientos y capacidades (Hartenian, 2003), al interaccionar las personas (Nonaka y Takeuchi, 1995). Se dan efectos de aprendizaje entre los miembros del equipo, haciéndoles más productivos (Hamilton et al., 2003).

El trabajo en equipo permite reducir los niveles jerárquicos y suprimir puestos que no aportan valor añadido, reduciendo los costes asociados (Pfeffer, 1998).

Según Delarue et al. (2008), basándose en referencias bibliográficas, el trabajo en equipo influye posi-

\footnotetext{
${ }_{1}^{1}$ Para Sundstrom et al. (1990) cada equipo está formado por un pequeño número de operarios que gestionan y desarrollan un segmento del proceso productivo, donde comparten responsabilidad por resultados concretos para su organización. Kozlowski y Bell (2003) los define como colectivos que existen para realizar tareas organizacionalmente relevantes, que mantienen un cierto grado de interdependencia tanto en términos de objetivos como de tareas, que gestionan y mantienen sus límites, estando inmersos en un contexto organizacional que restringe su actividad e influye sobre sus intercambios con otros equipos dentro de la organización.
} 
tivamente en la actitud del trabajador (involucración, compromiso y satisfacción), reduce ciertos resultados conductuales (p.e. la rotación en la empresa y el absentismo), permite mejorar la calidad (Dale y Boaden, 1994, Batt y Appelbaum, 1995), la innovación y la flexibilidad,que inciden positivamente en la productividad ${ }^{2}$ Asplund y Oksanen (2003) encuentran relación positiva del beneficio bruto de las empresas con el trabajo en equipo y la descentralización de las decisiones a nivel individual.

El trabajo en equipo genera el problema de no poder determinar el output individual de cada trabajador y entonces no poder recompensar individualmente según la productividad ofrecida, lo que puede generar conductas oportunistas por algunos de sus miembros (Alchian y Demsetz, op. cit.). Además, al responsabilizarse el operario de más actividades y su control, aumenta su estrés (Cully, 1999).

El trabajo en equipo se puede clasificar en "anti-taylorista" (sigue los sistemas socio-técnicos) o "neo-taylorista" (Pruijt, 2003). Niepce y Molleman (1996), Berggren (1993) y Pil y Fujimoto (2007) los diferencian, asociándolos a la producción reflexiva y la producción ajustada ${ }^{3}$. La producción ajustada requiere operarios con capacidad para detectar, analizar y resolver problemas (Puvamasvaran (2010).

La producción ajustada ofrece mayor competitividad a las empresas (MacDuffie, 1995; Womack et al., 1990; Melford, 2009), si bien los equipos en la producción ajustada son criticados por generar un trabajo monótono y repetitivo (Schouteten y Benders, 2004), una intensificación del trabajo, darse un fuerte control de la dirección (Garrahan and Stewart, 1992, Babson, 1993) y escaso empowerment a los trabajadores (Jackson y Martin, 1996; Lewchuck y Robertson, 1997).

A nivel empírico diversos estudios recogen la proporción muestral de empresas que incorporan la rotación de puestos y/o el trabajo en equipo. Respecto la rotación entre puestos, Osterman (1994) analizó en EEUU una muestra de establecimientos industriales, con al menos 50 trabajadores, aplicándose en un $55,6 \%$. En 1997 verificó que en el $55,5 \%$ de los nuevos establecimientos muestreados la penetración de la rotación entre puestos era al menos del $50 \%$, mientras que en las empresas con participación en 1992 y 1997 fue del 47,3\% (Osterman, 2000). En cambio, Gittleman et al. (1998) estimaron que la aplicaban el $24,2 \%$ en los establecimientos con al menos 50 trabajadores. La OCDE (1999) obtuvo en 1996 que el país europeo con mayor proporción muestral de centros de trabajo con rotación entre puestos era Suecia $(38 \%)$, mientras que en España era de los que menos (14\%). Kitson y Wilkinson (1999) estimaron que el $40 \%$ de los negocios en el Reino Unido aplicaban la rotación entre puestos. Friedrich et al. (1998) seleccionaron 14 países de Europa Occidental más Turquía, comprobrando que en Finlandia había mayor porcentaje muestral de organizaciones con rotación de puestos sistemática (33\%), mientras que España ocupaba la cuarta posición con el $23 \%$. Arundel et al. (2007) estimaron en la UE-15 que el $48,9 \%$ de los trabajadores encuestados rotaban entre sus puestos de trabajo. Posteriormente Kyzlinková el al. (2007) detectaron que en España la proporción muestral de trabajadores que rota entre puestos no supera el 40\%. García Olaverri y Huerta Arribas (1999) evidenciaron en una muestra de establecimientos industriales en España con al menos 50 trabajadores, que la rotación entre puestos estaba presente en el $43,8 \%$. Asimismo, Llorente $(2007,2013)$ ha constatado que la mayoría de empresas proveedoras de los fabricantes de automóviles localizadas en Cataluña incorporaban la rotación entre puestos al menos en grado medio.

Si se considera el trabajo en equipo, Osterman (1994) estimó en 1992 que en los establecimientos industriales con al menos 50 trabajadores de EEUU los disponían una proporción del $50,1 \%$. Posteriormente, encontró para los establecimientos que analizó en 1997, con al menos un $50 \%$ de penetración del trabajo en equipo, una proporción del 41,4\% (Osterman, 2000). Gittleman et al. (op. cit.) verificaron en establecimientos industriales con al menos 50 trabajadores que el $32 \%$ lo incorporaban. Lazear y Shaw (2007) estimaron

\footnotetext{
${ }^{2}$ Por ejemplo, lo verificaron Hamilton et al. (2003) y Eriksson (2003). Diversos autores han analizado la incidencia de las prácticas de alto rendimiento en la productividad, entre las que se incorporan la rotación y/o el trabajo en equipo (p.e. Huselid, 1995; MacDuffie, 1995; Ichiniowski et al. 1997; Ramsay et al., 2000; Capelli y Nuemark, 2001; Guthrie, 2001, 2008; Datta et al., 2005..En cambio, Birdi et al. (2008) no encontraron en el sector manufacturero del Reino Unido un impacto significativo del trabajo en equipo en la productividad.

${ }^{3}$ Para tales autores la producción ajustada se caracteriza porque el líder toma las decisiones, lo selecciona la empresa el trabajo está estandarizado, el tiempo ciclo es reducido, el operario contribuye a la mejora continua, y tiene escasa autonomía en planificar, organizar, establecer ritmos y métodos de trabajo. En cambio, en la producción reflexiva los operarios pueden participar en la toma de decisiones, se busca más la calidad de vida, el tiempo ciclo es elevado, los miembros del equipo eligen a su líder, y disponen de alta autonomía en planificar, organizar, establecer ritmos y métodos de trabajo. Para Benders et al. (1999) en la producción reflexiva las remuneraciones dependen de las habilidades, y las tareas a realizar son complejas, mientras que en la producción ajustada la remuneración es más uniforme (con fuerte peso de la antigüedad) y las tareas simples.
} 
que el $75 \%$ de las grandes empresas en EEUU se organizan con equipos, mientras que Lawler et al. (1992) obtuvo en las 1000 mayores empresas recogidas en Fortune una proporción del $47 \%$ y del $69 \%$ en Lawler et al. (1995).

Arundel et al. (op. cit) estimaron en la U.E.-15 que el $64,2 \%$ del personal trabaja en equipo. Kyzlinková et al. (op. cit.) identificaron en la UE-15 que los países más propensos a implantar el trabajo en equipo eran Reino Unido e Irlanda (con proporciones muestrales del $80,6 \%$ y $76 \%$, respectivamente). En cambio, España e Italia los que menos, con el $53,9 \%$ y $40,9 \%$. Previamente la OCDE (1999) recogió que en España el $30 \%$ de los centros de trabajo aplicaban el trabajo en equipo. Huerta Arribas et al. (2003) estimaron que el $56,6 \%$ de los establecimientos industriales en España con más de 50 trabajadores no incorporaba trabajo en equipo en 1996, mientras que en el 2007 fue del $60,9 \%$ (García Olaverri y Huerta Arribas, 2012). Llorente $(2007,2013)$ encontró también que en Cataluña la mayoría de proveedores directos de los fabricantes de automóviles no incorporan el trabajo en equipo y que cuando lo hacen tienden al de la producción ajustada. Riedmann et al. (2010) han evidenciado que la gran mayoría de empresas en España con trabajo en equipo, se organiza jerárquicamente.

\subsubsection{Delegación tareas indirectas en el operario}

A continuación se exponen determinadas prácticas que pueden delegarse al operario:

- Autonomía en planificar y organizar, establecer ritmos, y seleccionar métodos de trabajo. Suele asociarse con una menor intensidad en el trabajo (Kersley et al, 2006).
- Realizar autocontrol. Se responsabiliza de evaluar la conformidad del proceso o subproceso que desarrolla en su puesto de trabajo (Pérez-Fernández de Velasco, 1999), utilizando diversas herramientas estadísticas básicas ${ }^{4}$. Las empresas lo incorpora al implantar la gestión de calidad total.

- Participar en el análisis y solución de los defectos o anomalías encontrados, aplicando técnicas sencillas para el análisis de las causas ${ }^{5}$.

- Intervenir en la determinación del "estándar"6 y su posterior mejora, donde el conocimiento de los trabajadores es esencial (Cuatrecases y Olivella, 2006).

- Participar en la mejora continua. La mejora continua es un componente clave del TQM. Su aplicación ayuda a reducir el desperdicio y eliminar actividades sin valor añadido (Monden, 1983; Imai, 1986). Requiere de una participación activa de los trabajadores para mejorar las actividades (Jorgensen et al., 2004). Su aplicación posibilita incrementar la productividad o la eficiencia, la calidad, reducir el tiempo de fabricación (Grütter et al., 2002), la reducción de costes de producción (Terziovski y Shoal, 2000).

- Las " $5 S$ " son la base de la mejora continua, de la calidad total (Ugalde, 1998) y del TPM (Cuatrecasas, 2000). Posibilitan reducir el tiempo ciclo, los tiempos de preparación y cambios de serie, obtener más eficiencia, fiabilidad del equipo, calidad y productividad, mejorar las condiciones de trabajo, la seguridad y la ergonomía (Fortuny y Cuatrecasa, 2003, Chapman, 2006; Scott, 2005). Su uso se asocia con un impulso al perfomance en fabricación (Bayo-Moriones et al., 2010).

\footnotetext{
${ }^{4}$ Tales como la hoja de control, los histogramas, los diagramas de Pareto y los gráficos de control, entre otros.

${ }^{5}$ Se pueden incorporar además de las técnicas citadas anteriormente, otras para analizar mejor los problemas como los 5 por qué, los diagramas de flujo, diagramas de causas-efectos, diagramas de correlación, y también pueden aplicarse otras técnicas más complejas para buscar la solución, que requieren más trabajar en grupo y/o suelen ya aplicarlas los mandos intermedios o supervisores como son: la tormenta de ideas, los diagramas de afinidad, diagramas de relación, diagrama de árbol y diagrama de matriz. Árbol. El ciclo PDCA también es interesante utilizarlo.

${ }^{6}$ Para Adler y Cole (1995) la estandarización y los ciclos cortos de la producción ajustada permiten centrarse mejor en los detalles, ver antes los problemas y realizar mejor las labores de mejora continua. La estandarización estimula difundir las ideas de mejora continua a través de la organización.

${ }^{7}$ Iniciales de las palabras japonesas: Seiri (despejar), Seiton (orden), Seiso (limpieza), Seiketsu (normalizar) y Shitsuke (disciplina).
} 
Tabla 1. Diversa bibliografía que consideran las prácticas laborales seleccionadas

\begin{tabular}{|c|c|}
\hline Ítems & Bibliografía \\
\hline Autonomía del operario & $\begin{array}{l}\text { Berggren (1993), García Olaverri y Huerta Arribas (1999), Appelbaum et al. (2000), Bayo y Merino } \\
\text { (2002), Lorenz y Valery (2005), Arundel et al. (2007), Llorente (2007, 2008, 2014), Valeyre et al., } \\
\text { (2009), Urtason-Alonso et al. (2014) }\end{array}$ \\
\hline Autocontrol & $\begin{array}{l}\text { Karlsson y Ahlström (1996), García Olaverri y Huerta Arribas (1999),, Delbridge et al. (2000), Bayo } \\
\text { y Merino (2002); Llorente (2007, 2014), Lorenz y Valeyre (2005), Arundel et al. (2007), Valeyre et al. } \\
\text { (2009), Gutiérrez Broncano et al. (2011) }\end{array}$ \\
\hline Orden y limpieza & $\begin{array}{l}\text { García Olaverri y Huerta Arribas (1999), Trey (2003). Chapman (2006), Llorente }(2007,2008,2013) \text {, } \\
\text { Llorente (2007, 2013), Bayo-Moriones et al. (2008), Liker y Hosesus (2008, Bonavia y Marín (2010), } \\
\text { Naveen y Ganesh Kumar (2012), Llorente }(2007,2014)\end{array}$ \\
\hline Preparación de la maquinaria & García Olaverri y Huerta Arribas (1999), Llorente (2008), Llorente (2014) \\
\hline Mejora continua & $\begin{array}{l}\text { Imai (1986), Obloj et al. (1995), Delbridge et al. (2000), García Lorenzo y Prado (2002), Suzaki } \\
\text { (1993), Beauvallet y Houy (2007), Liker y Hosesus (2008), Bonavia y Marín (2010), Naveen y Ga- } \\
\text { nesh Kumar (2012), Llorente (2007, 2014) }\end{array}$ \\
\hline $\begin{array}{l}\text { Mantenimiento básico realizado por el } \\
\text { trabajador }\end{array}$ & $\begin{array}{l}\text { Delbridge et al. (2000), García Olaverri y Huerta Arribas (1999), Cuatrecasas (2000), Bullington } \\
\text { (2003); Suzaki (2004), Llorente (2007, 2014), Pil y Fujimoto (2007), }\end{array}$ \\
\hline Estandarización del trabajo & $\begin{array}{l}\text { Monden (1983), Ohno (1991); Suzaki (1993), Adler y Cole (1995), Ohno (1998), Spear y Bowen } \\
\text { (1999), Liker (2004), Cuatrecasas et al. (2008), Bonavia y Marín (2010) }\end{array}$ \\
\hline
\end{tabular}

Chung y Ross (1977) predicen que la satisfacción laboral y productividad es mayor cuando la ampliación del trabajo y el enriquecimiento del trabajo se dan conjuntamente para rediseñar el sistema de trabajo. Para Ichiniowki et al., (op. cit.) y Milgrom y Roberts (1995) cuando las prácticas laborales se incorporan conjuntamente como un sistema generan un impacto positivo superior, al darse complementariedades y sinergias entre las mismas.

Según Paent-Thirion (2007) España es de los países de la U.E. con menor autonomía de los trabajadores, lo que está en línea con Poutsma (2003), mientras que Alemania donde es mayor. Tangian (2007) analizó 30 países europeos, siendo España el país con menor flexibilidad funcional, mientras Dinamarca donde es superior. Llorente $(2007,2013)$ encontró que en Cataluña en los proveedores directos de los fabricantes de automóviles es intensa la implantación del orden y limpieza y el autocontrol, pero inferior el mantenimiento básico y el rediseño del trabajo.

Arundel et al. (op. cit) estimaron en la U.E.-15 que el $72,6 \%$ de los trabajadores tienen responsabilidad sobre el control de calidad y el $73,9 \%$ efectúan actividades de resolución de problemas. Para España García Olaverri y Huerta Arribas (1999) encontraron en los establecimientos industriales, a partir del estadísti- co media muestral, una intensidad media baja de los ítems el operario prepara las máquinas que utiliza, el mantenimiento de los equipos que utiliza y analizar datos, mientras que era reducida en planificar y organizar autónomamente el trabajo. Esos valores están en línea con los obtenidos por Llorente (2014) para las empresas de la industria automovilística en Cataluña.

A continuación se recogen diversos artículos de las consecuencias de incorporar las prácticas de alto rendimiento o de flexibilidad funcional.

Para Bresnahan et al. (2002) la incorporación de nuevos sistemas de trabajo es más probable que se asocie con exigir mayores niveles de educación en la plantilla.

Capelli y Neumark (2001) encontraron una relación positiva entre la incorporación del trabajo en equipo y el coste laboral por trabajador, pero era negativa con la rotación entre puestos, lo que también obtuvo Osterman (2006) al analizar los trabajadores "core" en una muestra de establecimientos en EEUU, mientras que Handel y Gittleman (2004) no encontraron influyeran para obtener unos mayores salarios.

Las prácticas de flexibilidad funcional influyen posi- 
tivamente en los resultados de la empresa (Huselid, 1995, Delaney y Huselid, 1996, Capelli y Neumark, 2001, Chan et al., 2004). Martínez et al. (2010) constataron para España que en las industrias auxiliar del automóvil, las telecomunicaciones y del software, la flexibilidad funcional incide positivamente en el resultado financiero de la empresa.

Michie y Sheehan (2001) detectaron que la flexibilidad funcional en combinación con los sistemas de trabajo de alto rendimiento parece mejorar el rendimiento financiero. Xue y Xu (2013) obtienen que la flexibilidad funcional favorece más al perfomance de la empresa que la flexibilidad numérica.

A partir de la exposición realizada, las hipótesis que se deciden plantear son:

- H1: Las modalidades de flexibilidad funcional tienen asociación positiva

- H2i: Igualdad en la medida de posición central de los ritmos de trabajo, nivel de stress y nivel de absentismo, segmentando según el grado de implantación de cada modalidad de flexibilidad funcional

- H3i: Igualdad en la medida de posición central de la productividad del trabajo, el margen de explotación y el peso del gasto de personal sobre las ventas, segmentando según el grado de implantación de cada modalidad de flexibilidad funcional

\subsubsection{Flexibilidad salarial}

Los sistemas retributivos variables se han convertido en un instrumento de las políticas de recursos humanos en la empresa, que se aplican como incentivos buscando una flexibilidad de costes, la comunicación de prioridades y motivar al personal para conseguir determinados resultados (Maella, 2012), así como el mejorar la productividad (Lazear, 2000; Origo, 2009; Gielen et al. 2010).

La estructura salarial está compuesta por el salario base y una parte variable o de compensación contingente, cuando se dispone, que se centra en los resultados individuales o de grupo (Gutiérrez Broncazo et al., 2011).

Para Martínez Sánchez et al. (2011) la flexibilidad salarial implica dejar una estructura salarial uniforme, estandarizada y fija, para adoptar sistemas retributivos basados en la evaluación del rendimiento del trabajador. En cambio, para Tsipouri et al. (2005) es la capacidad de la empresa para ajustar los costes salariales en función del perfomance de la empresa.
El sistema de retribuciones fijas dificulta los ajustes entre los costes y el precio final del producto (OCDE, 1987). Además, el sistema de remuneración calculado sobre la definición del puesto de trabajo es incompatible con una organización del trabajo donde las tareas se vuelven imprecisas y cambiantes (Maronnat-Geffroy, 2001).

Para Lawler (2000) los sistemas de remuneración variables proveen ventajas competitivas a la organización. El empleado debería recibir remuneraciones basadas en el valor que aporte a la organización y que esta comparta con el empleado una parte del perfomance que éste le aporta, para conseguir su compromiso.

Para determinar la parte variable pueden tenerse en cuenta diferentes criterios como son la remuneración ligada al rendimiento/productividad (puede incorporar el pago por pieza), la participación en los resultados de la empresa (Barth et al, 2008), planta (Olivella et al. (2008) o sección (Llorente, 2008), la adquisición de conocimientos, capacidades y habilidades (Stevens y Campion, 1994, Tremblay y Sire, 1999; Sweins y Kalmi, 2008), así como considerar los incentivos por grupos u objetivos conseguidos por el equipo del que se forma parte (De Varo, 2006, Llorente, 2008, Cuatrecasas et al., 2008) o incentivos ligados a mejoras en la calidad y eficiencia (Lawler et al., 2001).

La tendencia en Europa es reducir las formas más tradicionales de pagos variables como el pago por pieza, y sustituirlas por otras que incorporan esquemas de participación (van het Kaar y Grünell, 2001).

La remuneración por rendimiento puede estimular la productividad, por ejemplo, cuando hay información asimétrica sobre las habilidades de los trabajadores, y ese tipo de remuneración puede inducir a los trabajadores a ejercer la cantidad correcta de esfuerzo (Lazear, 2004), así como que aumenten los salarios (Ewing, 1996, Booth y Frank, 1999).

La flexibilidad financiera puede verse como un facilitador de determinadas prácticas de flexibilidad funcional, como el trabajo en equipo (Martínez Sánchez et al., 2011). Los incentivos colectivos se utilizan para fomentar la colaboración entre los trabajadores y buscar su compromiso en un mayor nivel de objetivos (Howard y Doughert, 2004). Además, facilitan que los miembros de los equipos transmitan sus conocimientos y obtengan aprendizaje mutuo, favoreciendo una mayor productividad (Che y Yoo, 2001). Los pagos basados en el perfomance del grupo, si están bien diseñados, incrementan la productividad (Bowie et al., 1993; Kruse, 1993b). 
El pago por beneficios es un sistema salarial directamente relacionado con los beneficios de la empresa en un período particular, compensándose por ello a los empleados en tal período (Kruse, 1992). Como recogen Long y Fang (2013) en la literatura hay 3 motivos para incorporar la participación en los beneficios: "a) sustituir parte de los componentes fijos salariales, b) atraer y retener capital humano relevante, c) aumentar la motivación de los empleados para alinearse con los objetivos de la empresa. Además, impulsa a los trabajadores a realizar un mayor esfuerzo, reducir costes y mejorar la calidad. Es preferible cuando el output no es fácil adscribirlo individualmente, y se busca la cooperación y productividad en el trabajo (Kruse, 1996). Una de las consecuencias de su implantación es la incerteza del importe que recibirá el trabajador por la misma (Wellbourne y Gómez Mejía, 1995).

Para Kalmi et al. (2005) el análisis de una muestra de empresas de Finlandia, Alemania, Holanda y Reino Unido considera genera dudas sobre la complementariedad entre la participación de los empleados en los beneficios y otros tipos de participación de los empleados. Además, obtienen la participación en los beneficios no incide en los resultados perfomance de las empresas, lo que sigue la lógica obtenida por Poutsma et al., (2003).

Si se desea que los trabajadores adquieran múltiples habilidades, es aconsejable un sistema de remuneración que tenga en cuenta su consecución (Cordery 1989, Flood et al., 2008). Tal remuneración es previsible que se adopte especialmente para los trabajadores del conocimiento (Ledford, 1995).

Para Karlsson y Áhlström (1995) la producción ajustada implica introducir cambios en el sistema de remuneración, incorporando en el salario variable las mayores responsabilidades del trabajador y del equipo al que se adscribe. Karlsson y Áhlström (1996) describen la remuneración en la producción ajustada como un sistema de remuneración con una parte fija basada en las competencias y la responsabilidad asumida por el trabajador en el grupo, más un bono basado en la productividad, la calidad y número de pedidos entregados a tiempo por el equipo. Para Olivella et al. (2008) la compensación en la producción ajustada debería considerar el perfomance de la planta, el perfomance del equipo y los skills de los trabajadores, recogiendo estos dos últimos de Sodenkamp et al. (2005).

En la práctica, el salario de base fija es mayoritario en la U.E.-15, reflejando Lorenz y Valeyre (2005) que lo tienen el $92,8 \%$ de los trabajadores, siendo elevado incluso en las organizaciones con producción ajustada $(91,9 \%)$ o en aprendizaje $(93,2 \%)$. En el caso de la industria metalúrgica en España, para Novella y Aluja (2008) "la estructura de pago fijo es la tradicional. Un sueldo básico determinado por el tiempo de trabajo y la clasificación de puestos, unos complementos basados en la antigüedad, incentivos productivos, la puntualidad y suplementos del puesto de trabajo debido a circunstancias especiales (p.e. toxicidad, peligrosidad)" . Huerta el al. (2003) verifican que el porcentaje de empresas industriales españolas con al menos 50 trabajadores que utilizan los incentivos a la productividad es próxima a la mitad de las empresas muestreadas y supera a las empresas de Alemania, Inglaterra y Australia ${ }^{8}$

España es de los países de la U.E. donde menos se aplica la participación en los beneficios (Poutsma, 2001; Welz y Fernándes-Macías, 2007). En 1996 las empresas industriales españolas con al menos 50 trabajadores un $13 \%$ disponían de incentivos de calidad y el $18,3 \%$ incentivos vinculados a los resultados del establecimiento o la empresa (Huertas et al., 2003); Bayo-Moriones (2003) obtuvo para la Metalurgia que el $18,3 \%$ lo utilizaban y en el de Material de Transporte el $10,2 \%$. Asimismo, Bonavia y Marín (2010) en el sector cerámico español obtuvo una media de remuneración variable del $9,9 \%$ y Llorente $(2007,2013)$ en la industria auxiliar de Cataluña encontraron en los operarios que la parte salarial variable sobre el total era reducida.

Lawler y Mohr (2003) analizaron en el 2002 las 1000 empresas de Fortune y encontraron que solo el $7 \%$ compensaban sus habilidades o conocimientos, en el $24 \%$ por trabajar en equipo y por participar en beneficios en el $27 \%$. En Irlanda Flood et al. (2005) estimaron que el $26,6 \%$ se consideran los conocimientos y habilidades en el salario variable. Llorente (2013) obtuvo en Cataluña que tal modalidad la aplicaban cerca del $10 \%$ de las empresas que son proveedores directos de los fabricantes de automóviles.

La implantación de los sistemas de pago variables han aumentado recientemente (Welz y Fernándes-Macías, 2008).

Ciertos estudios apuntan a una relación positiva entre los sistemas de remuneración variable y la productividad $^{9}$. En el caso de incentivos individuales ciertos autores así lo consideran (Mitchell et al., 1990; Parent,

\footnotetext{
${ }^{8}$ Huerta Arribas et al. (2003) recogen diversos estudios realizados en otros países para sustentarlo.

9 Por ejemplo: Cable y Wilson (1989), Wadhwani y Wall (1990), Doucouliagos (1995), OECD (1995) y Ewing (1996).
} 
2002). En cambio, otros arrojan serias dudas sobre estos resultados (p.e., Robinson y Wilson, 2006; Cox, 2005).

El aplicar esquemas de remuneración por rendimiento se ha verificado que incrementan los niveles de productividad en el trabajo en el sector del metal en Italia (Origo, 2009) y en las empresas holandesas (Gielen et al., 2006).

La implantación de participación en los beneficios pueden ayudan a mejorar la productividad (Long, 1997; Kato y Morishima, 2003, Robinson y Wilson, op. cit., Piekkola, 2005). El meta-análisis realizado por Weitzman y Kruse (1990) recoge que el impacto de participar en los beneficios respecto la productividad es positivo. La diversidad de artículos que recogen Kruse (1993a), Doucouliagos (1995), D’Art y Turner (2004), y Blasi et al (2010), verifican también una relación positiva. Asimismo, Cahuc y Dormont (1997) encontraron en las empresas francesas un leve aumento de la productividad por la participación en los beneficios. Jana y Petr (2013), en su análisis bibliométrico, obtienen que la mayoría de estudios ofrecen un impacto positivo o neutro de la participación en los beneficios en la productividad o la rentabilidad. Long y Fang (2013) verificaron en Canadá que hay relación entre los programas de participación en beneficios y el incremento de productividad, pero sólo en establecimientos que utilizan el trabajo en equipo.

Ridemann et al. (2010) en su estudio sobre las empresas europeas, obtienen que en la U.E-27 aproximadamente el $35 \%$ de los establecimientos de la industria con al menos 10 trabajadores tienen que en el salario del personal incide el perfomance individual o del grupo. Asimismo, los establecimientos en España incorporan en algo más del $30 \%$ el salario variable individual por perfomance en España, mientras que el salario por perfomance del grupo, prácticamente el $20 \%$. Además, en las empresas del sector privado el $14 \%$ de los establecimientos de la U.E. incorporan el pago por beneficios para todos los trabajadores y un $10 \%$ en el caso de los establecimientos de la industria y energía. Por países, en España aproximadamente del $12 \%$ del total de establecimientos con al menos 10 trabajadores lo aplican, con una proporción ligeramente superior en las grandes empresas. En cambio, Francia es donde más se utiliza (cerca del $28 \%$ ), siendo claramente las grandes empresas quienes más. Las razones que destacan para implantar el pago por beneficios son la mejora de la motivación de la plantilla y aumentar la productividad.

McNabb y Whitfield (1998) recogen diferente bibliografía, observando que no hay resultados concluyentes del impacto de los sistemas de participación financiera de los empleados en el perfomance económico de las empresas. Posteriormente, tampoco encontraron relación entre la participación en los beneficios y los ingresos de los trabajadores (McNabb y Whitfield, 2007), mientras que sí la obtuvo Handel y Gittleman (2004) en establecimientos de USA. En la literatura no hay consenso sobre sus efectos en los ingresos de los trabajadores, pues para ciertos autores su incidencia es positiva, para otros es neutral e incluso algunos consideran que puede ser negativa (Long y Fang, 2012).

Los sistemas salariales que tienen en cuenta las habilidades del personal son más probables que satisfagan necesidades psicológicas, mejorando la motivación en el trabajo, al facilitar a los trabajadores estar en puestos de trabajo más enriquecidos. (Murray y Gerhart, 1996).

Las empresas que incorporan la producción flexible, con trabajo en equipo, rotación entre puestos y mayor delegación de responabilidades precisan de una continua inversión en capital humano (Asplund y Oksanen, op. cit.).

\section{Análisis empírico}

\subsection{Metodología}

\subsubsection{Estructura del cuestionario}

El cuestionario consta de diversos bloques según los ámbitos que se analizan:

- Aspectos generales de la empresa: volumen de ventas, volumen de la plantilla, nivel de estudios de la plantilla.

- Prácticas laborales aplicadas. Se incorporan ítems sobre diversas tareas/funciones que el operario puede asumir, la rotación entre puestos, la rotación entre secciones, el trabajo en equipo, la proporción del salario variable sobre el salario total y las modalidades de salarios variables.

- Características de los puestos de trabajo: monotonía, tareas cortas y repetitivas, ritmos de trabajo y nivel de estrés.

- Grado de absentismo.

Previamente a la recogida de datos se realizó un pretest con académicos y profesionales del sector para conocer su opinión sobre los ítems considerados ini- 
cialmente. Posteriormente también una prueba piloto a presidentes comités de empresa y responsables de recursos humanos de 5 empresas para incorporar posibles mejoras, a partir de sus tasas de respuesta y comentarios.

La mayoría de las variables son cualitativas, destacando las medidas en escala ordinal, con una escala de Likert de valores 0 a 10 , que permiten dar una medida del grado de implantación del ítem a analizar. La dificultad o imposibilidad de obtener datos numéricos de ciertos ítems para la mayoría de empresas, tuvo como consecuencia el tener que adoptar alternativamente la utilización de variables ordinales u nominales.

\subsubsection{Ficha técnica de la encuesta:}

La población se constituye por las empresas medianas y grandes del sector metal localizadas en Cataluña. La ficha técnica es:

Tabla 2. Ficha técnica

\begin{tabular}{|c|c|}
\hline Alcance & Comunidad autónoma de Cataluña \\
\hline Población & $\begin{array}{l}\text { Empresas del sector metal con al menos } \\
100 \text { trabajadores }\end{array}$ \\
\hline $\begin{array}{l}\text { Elementos de } \\
\text { muestreo }\end{array}$ & $\begin{array}{l}\text { Los presidentes de los comités de em- } \\
\text { presa }^{10} \text {. Si desconocían algunas cues- } \\
\text { tiones, nos dirigimos al director de per- } \\
\text { sonal o bien de producción. }\end{array}$ \\
\hline Tipos de preguntas & $\begin{array}{l}\text { Mayoritariamente son cualitativas de } \\
\text { escala ordinal, buscando la opinión en } \\
\text { una escala de } 0 \text { a } 10 \text {. Minoritariamente } \\
\text { también hay cuantitativas y nominales } \\
\text { dicotómicas. }\end{array}$ \\
\hline $\begin{array}{l}\text { Fecha del trabajo } \\
\text { de campo }\end{array}$ & Año 2008 y revisado en el 2009 \\
\hline Muestra final & $\begin{array}{l}\text { El tamaño muestral es de } 100 \text { empresas, } \\
\text { que supone un } 41,5 \% \text { de la población. }\end{array}$ \\
\hline Error muestral & $\begin{array}{l}\text { Tomando la expresión de la estimación } \\
\text { de las proporciones poblacionales, un } \\
\text { nivel de confianza del } 95 \% \text { y escoger la } \\
\text { máxima holgura, el margen de error de } \\
\text { muestreo de la estimación fue del } 7,5 \\
\% \text {. }\end{array}$ \\
\hline
\end{tabular}

\subsubsection{Metodología}

En el tratamiento estadístico de los datos se ha efectuado:

- Análisis descriptivo univariante según la naturaleza de cada variable.

- Tablas de contingencia bidimensionales y múltiples para las variables ordinales y dicotómicas seleccionadas conjuntamente, obteniendo las respectivas frecuencias absolutas conjuntas y proporciones muestrales.

- Contrastar la intensidad de asociación lineal mediante el coeficiente de correlación de Pearson para pares de variables cuantitativas y el de Spearman para variables ordinales o bien si una es ordinal y la otra cuantitativa.

- Contrastar la hipótesis nula de independencia entre cada par de atributos dicotómicos con el estadístico chi cuadrado (con corrección de Yates) o bien realizar el test exacto de Fisher si los valores esperados mayores a 5 son inferiores al $80 \%$. Si se rechaza tal hipótesis nula entonces se mide la intensidad de asociación bivariante mediante los estadísticos: $Q$ de Yule (si las variables son dicotómicas) y $\mathrm{V}$ de Cramer (si hay más de dos categorías en uno de los atributos).

- Contrastar la hipótesis nula de igualdad en el valor de la medida de tendencia central de la variable cuantitativa u ordinal considerada, según la variable de agrupación, frente a la alternativa de que hay diferencias significativas. Se aplican las pruebas $U$ de Mann Whitney o bien Kruskal y Wallis.

- Análisis Homals para reducir la dimensionalidad, las principales variables que las explican, y obtener relaciones entre más de dos variables. Se aplica un análisis cluster sobre los puntos objeto obtenidos para establecer una tipología de empresas.

\subsection{Explotación de los datos ${ }^{11}$}

\subsubsection{Análisis univariante}

La "rotación entre puestos" suele estar implantada intensamente (mediana=7), mientras que la "rotación entre secciones" es reducida (mediana=2). La mayoría

\footnotetext{
${ }^{10}$ Se agradece a Pere Colell de la Federación del Industria de CCOO Cataluña y Juán Antonio Pasadas de MCA UGT Cataluña la colaboración recibida para contactar con los responsables sindicales de las empresas.

${ }^{11}$ Una primera presentación de parte de los datos se realizó en una comunicación presentada por el Autor (2013) a un Congreso de Economía.
} 
de las empresas no incorporan el "trabajo en equipo en el área de producción" (mediana=0) y sólo el $16 \%$ en grado alto (valor al menos de 7). Por tanto, mayoritariamente no aprovechan las ventajas señaladas que ofrece el trabajar en equipo en el área de producción.
Los equipos con personal de diferentes departamentos para solucionar problemas en la mayoría de empresas están más instalados, si bien sólo de forma intensa en el $14 \%$ de las empresas.

Tabla 3. Rotación entre puestos, entre secciones, y tipos de equipos. Estadísticos

\begin{tabular}{|c|c|c|c|c|c|}
\hline Variable & Media & Desv. Est. & Mediana & Rango intercuartílico & Valor $\geq 7$ \\
\hline Rotación entre puestos & 5,6 & 2,6 & 6 & 3 & $9 \%$ \\
\hline Rotación entre secciones & 2,9 & 2,5 & 2 & 1 & $38 \%$ \\
\hline $\begin{array}{l}\text { Trabajo en equipo área produc- } \\
\text { ción }\end{array}$ & 2,3 & 3,1 & 0 & 5 & $16 \%$ \\
\hline $\begin{array}{l}\text { Equipos con personal diferentes } \\
\text { dptos. para solucionar proble- } \\
\text { mas }\end{array}$ & 3,12 & 2,8 & 3 & 5 & $14 \%$ \\
\hline Equipos de mejora voluntarios & 2,5 & 2,9 & 1,5 & 5 & $12 \%$ \\
\hline Equipos de mejora obligatorios & 1,3 & 2,2 & 0,0 & 2,0 & $14 \%$ \\
\hline
\end{tabular}

La tabla 4 refleja una elevada delegación al operario de que ordene y limpie su puesto de trabajo, junto al autocontrol y la estandarización del trabajo (ambos son base de las $5 \mathrm{~S}$ ). Además está bastante incorporado que el operario participe en la mejora continua y prepare la maquinaria. Se puede considerar significativa la participación del operario en buscar las causas de los defectos (utilizando herramientas estadísticas como los gráficos de Pareto, el SPC o los diagramas causa-efecto) y después darles solución. En cambio, en menor medida el operario realiza el mantenimiento básico, las pequeñas reparaciones y programa la maquinaria que utiliza, siendo escasa su participación en la elaboración de los estándares y posterior mejora. Asimismo, en la mayoría de las empresas hay un reducido rediseño del trabajo, como se observa en los ítems: autonomía de los operarios para planificar y organizar su trabajo, establecer sus ritmos de trabajo y escoger los métodos para realizar su trabajo, no superando ninguno de los tres ítems el valor 3 para la mediana y no llegan a tener valores altos en tales ítems la cuarta parte de las empresas. Si bien la mayoría de empresas tiene el trabajo muy estandarizado, sin embargo, no llegan al $10 \%$ las que sus operarios participan intensamente en la elaboración del trabajo estandarizado y en su posterior mejora.

Tabla 4. Prácticas laborales. Grado de implantación. Estadísticos

\begin{tabular}{|l|c|c|c|c|c|}
\hline \multicolumn{1}{|c|}{ Variables } & Media & D.E. & Me & $\begin{array}{c}\text { Rango intercuar- } \\
\text { tílico }\end{array}$ & Valor $\geq 7$ \\
\hline $\begin{array}{l}\text { Autonomía del operario en planificar y organizar el tra- } \\
\text { bajo }\end{array}$ & 3,3 & 2,7 & 3,0 & 4,0 & $15,0 \%$ \\
\hline $\begin{array}{l}\text { Autonomía del operario en establecer los ritmos de } \\
\text { trabajo }\end{array}$ & 3,4 & 2,9 & 3,0 & 3,0 & $17,0 \%$ \\
\hline $\begin{array}{l}\text { Autonomía del operario en establecer los métodos del } \\
\text { trabajo }\end{array}$ & 2,7 & 2,0 & 2,0 & 5,0 & $12,0 \%$ \\
\hline
\end{tabular}




\begin{tabular}{|c|c|c|c|c|c|}
\hline Participación del operario en la mejora continua & 4,1 & 2,6 & 4,0 & 4,0 & $20,0 \%$ \\
\hline Autocontrol & 6,2 & 2,7 & 7,0 & 2,0 & $57,0 \%$ \\
\hline Operario ordena y limpia el área de trabajo & 7,8 & 2,0 & 8,0 & 1,0 & $76,0 \%$ \\
\hline Operario prepara la maquinaria & 5,7 & 3,1 & 6,0 & 4,0 & $47,0 \%$ \\
\hline Operario programa la maquinaria & 3,7 & 3,4 & 3,0 & 7,0 & $30,0 \%$ \\
\hline Operario realiza el mantenimiento básico & 3,9 & 3,3 & 4,0 & 5,7 & $25,0 \%$ \\
\hline Operario realiza pequeñas reparaciones & 3,5 & 3,0 & 4,0 & 1,0 & $23,0 \%$ \\
\hline Operario busca el origen de los defectos & 4,7 & 3,0 & 5,0 & 5,0 & $33,0 \%$ \\
\hline Operario soluciona solo los defectos & 4,5 & 2,5 & 5,0 & 4,0 & $22,0 \%$ \\
\hline Operario soluciona con otros los defectos & 5,6 & 2,7 & 5,5 & 3,7 & $37,0 \%$ \\
\hline Estandarización del trabajo & 7,0 & 2,4 & 7,0 & 1,8 & $63,0 \%$ \\
\hline Operario participa en elaborar el trabajo estandarizado & 2,3 & 2,6 & 1,0 & 4,0 & $6,0 \%$ \\
\hline $\begin{array}{l}\text { Operario participa en la mejora del trabajo estandariza- } \\
\text { do }\end{array}$ & 2,5 & 2,6 & 2,0 & 5,0 & $9,0 \%$ \\
\hline
\end{tabular}

Una de las dificultades relevantes, expuestas por algunas direcciones de recursos humanos en entrevistas telefónicas, para aumentar la delegación en sus operarios, es que precisarían tener un personal con mayor nivel de estudios, ya que la media muestral de la distribución del personal con estudios bajos es del $56 \%$.

A continuación se analizan en las empresas que incorporan el trabajo en equipo ( 45 empresas), las proporciones muestrales con valoración al menos de 4 (medio-baja) y al menos 7 (alta) (ver tablas 5 y 6). Se verifica que el trabajo en equipo generalmente incorpo- ra diversos aspectos de la producción ajustada, puesto que en la mayoría de empresas que la han implantado sus operarios realizan un elevado autocontrol, ordenan y limpian sus puestos de trabajo, rotan entre puestos de trabajo, y tienen el trabajo estandarizado. Además, el tiempo ciclo es reducido. En cambio, el operario debería en mayor medida buscar el origen de los defectos y solucionarlos, así como realizar más el mantenimiento básico y las pequeñas reparaciones (incorporando el TPM), participar en la mejora continua, la elaboración del estándar y mejora del mismo.

Tabla 5. Empresas que incorporan el trabajo en equipo en el área de producción. Prácticas laborales (I)

\begin{tabular}{|l|l|l|l|l|l|l|l|}
\hline & $\begin{array}{l}\text { Rotación en } \\
\text { puestos }\end{array}$ & Autocontrol & $\begin{array}{l}\text { Ordenar y limpiar } \\
\text { puestos trabajo }\end{array}$ & $\begin{array}{l}\text { Operario hace } \\
\text { manto. básico }\end{array}$ & $\begin{array}{l}\text { P e que ñ a s } \\
\text { reparaciones }\end{array}$ & $\begin{array}{l}\text { Participa en } \\
\text { la } \\
\text { mejora } \\
\text { continua }\end{array}$ & $\begin{array}{l}\text { P r e p a r a } \\
\text { maquinaria }\end{array}$ \\
\hline Valoración $\geq 4$ & $43(95,6 \%)$ & $38(84,5 \%)$ & $42(93,3 \%)$ & $25(55,6 \%)$ & $22(48,9 \%)$ & $33(73,3 \%)$ & $34(65,6 \%)$ \\
\hline Valoración $\geq 7$ & $23(51,1 \%)$ & $25(55,6 \%)$ & $35(77,7 \%)$ & $12(26,7 \%)$ & $8(17,8 \%)$ & $14(31,1 \%)$ & $20(44,4 \%)$ \\
\hline
\end{tabular}


Tabla 6. Empresas que incorporan el trabajo en equipo en el área de producción. Prácticas laborales (II)

\begin{tabular}{|c|c|c|c|c|c|c|c|c|}
\hline & $\begin{array}{l}\text { Programa } \\
\text { maquinaria }\end{array}$ & $\begin{array}{l}\text { Busca ori- } \\
\text { gen defec- } \\
\text { tos }\end{array}$ & $\begin{array}{c}\text { Analiza } \\
\text { datos }\end{array}$ & $\begin{array}{l}\text { Soluciona } \\
\text { solo los de- } \\
\text { fectos }\end{array}$ & $\begin{array}{l}\text { Sol. con } \\
\text { otros los } \\
\text { defectos }\end{array}$ & $\begin{array}{c}\text { Estandarización } \\
\text { del trabajo }\end{array}$ & $\begin{array}{l}\text { Part. Elab- } \\
\text { orar traba- } \\
\text { jo estand. }\end{array}$ & $\begin{array}{c}\text { Part. Mejora } \\
\text { Trabajo est. }\end{array}$ \\
\hline $\begin{array}{c}\text { Valoración } \\
\quad \geq 4\end{array}$ & $9(20,0 \%)$ & $11(24,4 \%)$ & $\begin{array}{c}25 \\
(55,5 \%) \\
\end{array}$ & $29(64,4 \%)$ & $\begin{array}{c}35 \\
(77.7 \%) \\
\end{array}$ & $42(93,3 \%)$ & $14(31,1 \%)$ & $17(37,8 \%)$ \\
\hline $\begin{array}{c}\text { Valoración } \\
\quad \geq 7\end{array}$ & $16(35,6 \%)$ & $22(48,9 \%)$ & $\begin{array}{c}12 \\
(26,7 \%)\end{array}$ & $9(20,0 \%)$ & $\begin{array}{c}15 \\
(33,3 \%)\end{array}$ & $30(66,7 \%)$ & $2(4,4 \%)$ & $4(8,9 \%)$ \\
\hline
\end{tabular}

En las empresas con trabajo en equipo en el área de producción 37 ofrecen datos del ítem "Personal con estudios bajos/Plantilla", al contestar a la variable nivel de estudios, y la mediana de su distribución es del $62,5 \%$. Este valor elevado puede dificultar la eficacia del trabajo en equipo, pues exige un enriquecimiento del trabajo, implantándose una mayor delegación de tareas indirectas en el operario. Por tanto, ello podría generar que la al aplicar la producción ajustada se obtuvieran menores resultados e imposibilitar especialmente la producción reflexiva, ya que en este caso el trabajador debe de asumir un elevado enriquecimiento vertical, con un alto rediseño del trabajo, exigiendo una cualificación del operario mayor al de la producción ajustada.

Para analizar la flexibilidad salarial del operario, se ha escogido como indicador el "porcentaje del salario variable sobre el salario total", dando su distribución una media muestral de $17,7 \%$ y coeficiente de variación de valor 0,94 , siendo la media recortada al $5 \%$ el $15,8 \%$, valor que consideramos es bajo.

De las modalidades de remuneración variable consideradas la mayor frecuencia relativa muestral corresponde a "Rendimiento/productividad"12 (34\%) (ver tabla 7), seguido por los "incentivos de equipo". En cambio es muy reducida al considerar los resultados de la planta o sección.
Tabla 7. Algunas modalidades de remuneración variable para los operarios. Proporción muestral

\begin{tabular}{|l|c|}
\hline & Porcentaje \\
\hline Rendimiento / Productividad & $34,0 \%$ \\
\hline Calidad conseguida & $10,0 \%$ \\
\hline Incentivos por cumplir objetivos del equipo & $12,0 \%$ \\
\hline Resultado de la sección & $1,0 \%$ \\
\hline Resultado de la planta & $3,0 \%$ \\
\hline Resultado de la empresa & $15,0 \%$ \\
\hline Capacidades/Habilidades/Conocimientos & $13,0 \%$ \\
\hline
\end{tabular}

En las empresas con trabajo en equipo en el área de producción en grado alto, el $26,7 \%$ incorporan en el salario variable la modalidad cumplir los objetivos del equipo.

\subsubsection{Medidas de asociación y tablas de contingencia}

Al contrastar la asociación entre los ítems de rediseño del trabajo, mediante el coeficiente rho de Spearman, la intensidad de asociación entre la "Autonomía para planificar y organizar el trabajo" y la "Autonomía en establecer los ritmos de trabajo" es significativa e intensa (rho $=0,747, p$-valor $=0,000$ ), incidiendo que la mayoría de empresas disponen de valores reducidos de ambos ítems simultáneamente. Se verifica también una intensidad de asociación medio-alta entre la "Autonomía en

\footnotetext{
${ }^{12}$ De la totalidad de empresas que aplican esta modalidad, el 64,7\% tienen implantado un sistema de primas por cantidad producida. En el cuestionario se separaron como ítems "sistema de primas por cantidad producida" y "Rendimiento/Productividad" obteniéndose unas proporciones maestrales del $36 \%$ y el $27 \%$, respectivamente. En la tabla los consideramos a ambos como un único ítem.
} 
los ritmos del trabajo" y la "Autonomía en los métodos de trabajo" (rho $=0,613$, p-valor $=0,000$ )" y de grado medio entre la "Autonomía para planificar y organizar el trabajo" y la "Autonomía en los métodos en el trabajo" (rho $=0,521, p$-valor $=0,000$ ).

La intensidad de asociación entre los diferentes tipos de delegación de tareas indirectas al operario en su mayoría es significativa y de grado medio-bajo (tabla 8). "Participar en la mejora continua" es el ítem de menor asociación con la mayoría del resto de ítems.

En cambio, destacan el grado de asociación de los ítems "Prepara la maquinaria" y "Realiza pequeñas reparaciones" con el resto de ítems de la tabla 9. Se supera el valor rho=0,6 (medio-alto) en los pares "Prepara maquinaria" y "Programa maquinaria", así como entre "Busca el origen de los defectos" y "Soluciona los solo los defectos".

Tabla 8. Rho de Spearman entre prácticas laborales (I).

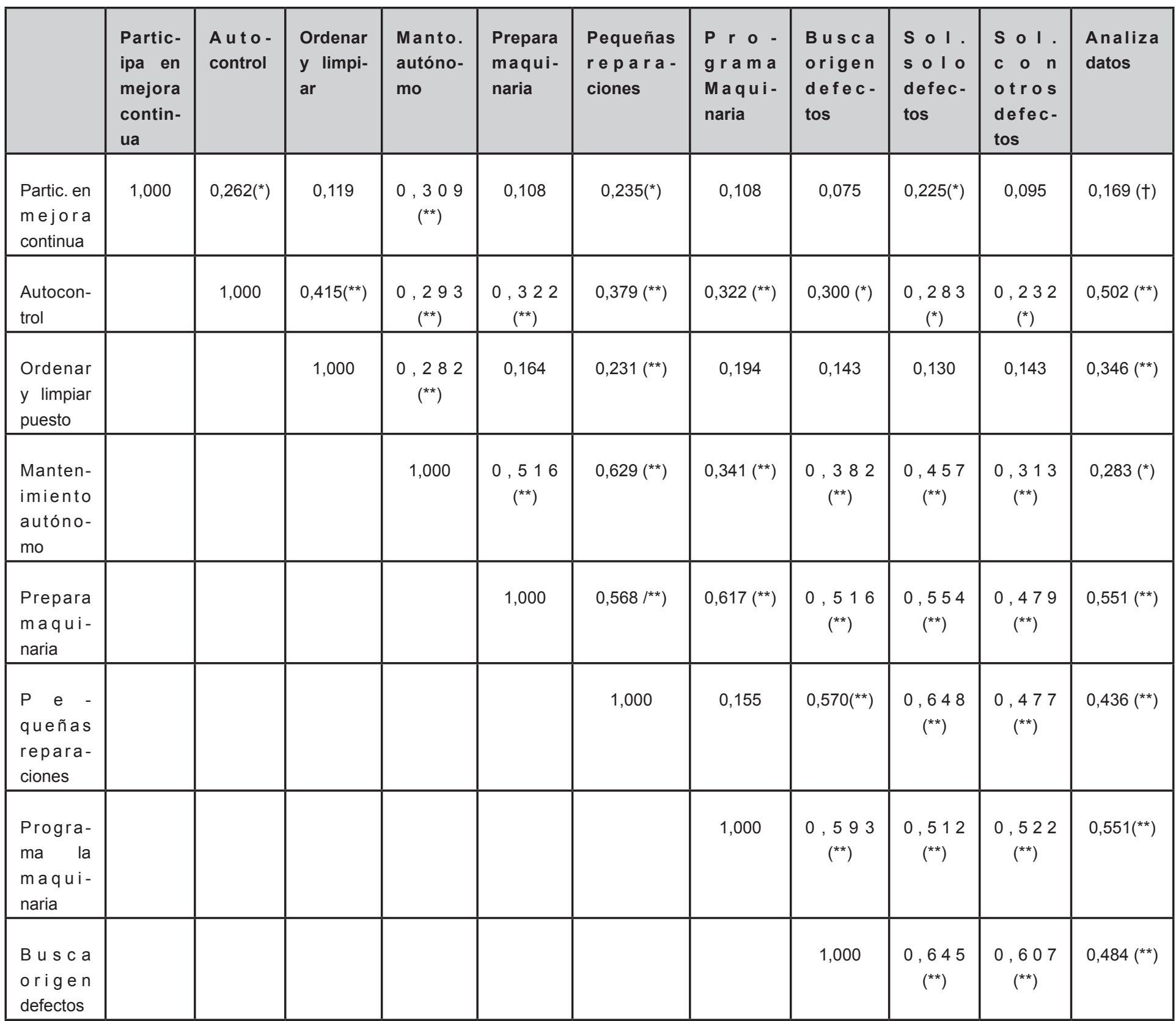




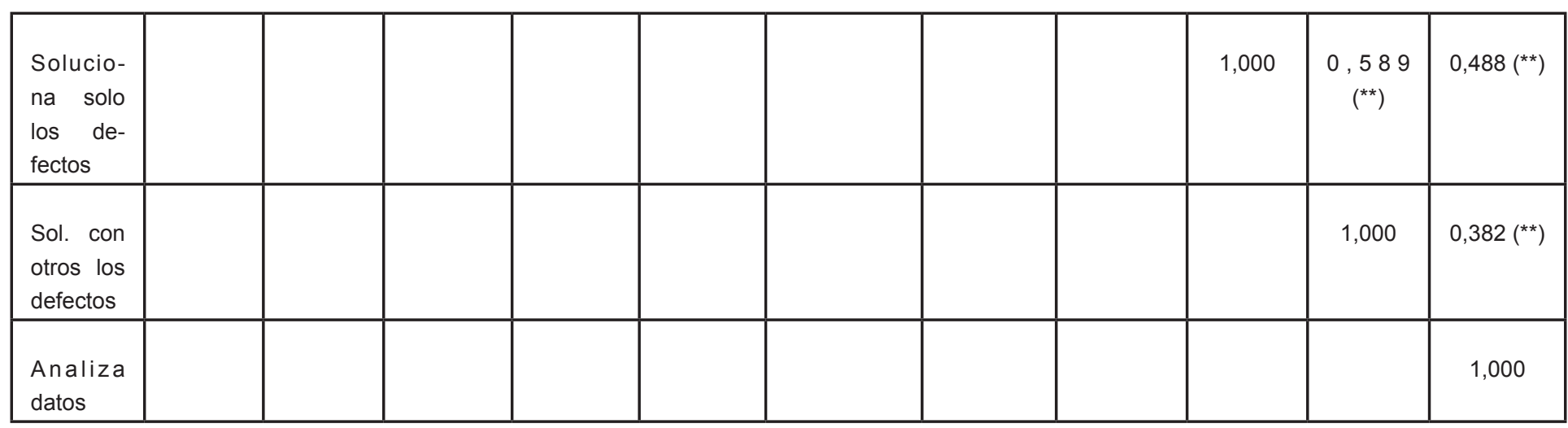

(†) $p<0,1 ; \quad\left(^{*}\right) p<0,05 ;\left(^{* *}\right) p<0,01$

Los ítems "Participa el operario en la elaboración del trabajo estandarizado" y "Participa el operario en la mejora del trabajo estandarizado" tienen asociación significativa al $5 \%$ con el resto de prácticas laborales consideradas en la tabla 9, con intensidad baja o media-baja. En cambio, carecen de asociación significativa con los ítems "Rotación entre puestos" y "Trabajo en equipo en el área de producción".

Tabla 9. Rho de Spearman entre prácticas laborales (II).

\begin{tabular}{|c|c|c|c|c|c|c|c|c|c|c|}
\hline & $\begin{array}{l}\text { Partic- } \\
\text { ipa en } \\
\text { mejora } \\
\text { continua }\end{array}$ & $\begin{array}{l}\text { A u t o - } \\
\text { control }\end{array}$ & $\begin{array}{l}\text { Manto. } \\
\text { autóno- } \\
\text { mo }\end{array}$ & $\begin{array}{l}\text { Prepara } \\
\text { maqui - } \\
\text { naria }\end{array}$ & $\begin{array}{l}P \quad \text { e - } \\
\text { queñas } \\
\text { repara- } \\
\text { ciones }\end{array}$ & $\begin{array}{l}P r o- \\
g r a m a \\
M \text { aqui- } \\
\text { naria }\end{array}$ & $\begin{array}{l}\text { B u s c a } \\
\text { origen } \\
\text { defectos }\end{array}$ & $\begin{array}{l}\text { Sol. solo } \\
\text { defectos }\end{array}$ & $\begin{array}{l}\text { Sol. con } \\
\text { o t r o s } \\
\text { defectos }\end{array}$ & $\begin{array}{l}\text { Analiza } \\
\text { datos }\end{array}$ \\
\hline $\begin{array}{l}\mathrm{P} \text { a } \mathrm{rt} \text {. } \\
\text { elab. tra- } \\
\text { bajo es- } \\
\text { tándar }\end{array}$ & $0,207\left(^{*}\right)$ & $0,294\left(^{* *}\right)$ & $0,285\left(^{(* *}\right)$ & $0,274\left(^{* *}\right)$ & $0,455\left(^{(* *}\right)$ & $0,255\left(^{* *}\right)$ & $0,329\left(^{* *}\right)$ & $0,398\left(^{* *}\right)$ & $0,255\left(^{* \star}\right)$ & $0,274\left({ }^{* *}\right)$ \\
\hline $\begin{array}{l}\mathrm{Part} \\
\mathrm{mejora} \\
\text { trabajo } \\
\text { estandar. }\end{array}$ & $0,435\left(^{* *}\right)$ & $0,318\left(^{* *}\right)$ & $0,298\left({ }^{* *}\right)$ & $0,273\left(^{* *}\right)$ & $0,419\left({ }^{* *}\right)$ & $0,236\left(^{* *}\right)$ & $0,255\left(^{(*}\right)$ & $\left.0,367^{(* *}\right)$ & 0,155 ) & $0,245\left(^{*}\right)$ \\
\hline
\end{tabular}

(†) $p<0,1 ; \quad\left(^{*}\right) p<0,05 ;\left({ }^{* *}\right) p<0,01$

La rho de Spearman entre los ítems que recogen el rediseño del trabajo y las variables de delegación de tareas indirectas en el operario (tabla 10 y 11), refleja que cuando hay asociación significativa entonces es baja o medio-baja, siendo superior con "solucionar los defectos". 
Tabla 10. Rho de Spearman entre el rediseño trabajo y otras prácticas laborales (I)

\begin{tabular}{|c|c|c|c|c|c|c|c|c|c|c|c|}
\hline & $\begin{array}{l}\text { Partic- } \\
\text { ipa en } \\
\text { mejora } \\
\text { contin- } \\
\text { ua }\end{array}$ & $\begin{array}{l}\text { A u t o - } \\
\text { control }\end{array}$ & $\begin{array}{l}\text { Orde - } \\
\text { nar y } \\
\text { limpiar }\end{array}$ & $\begin{array}{l}\text { Manto. } \\
\text { autóno- } \\
\text { mo }\end{array}$ & $\begin{array}{l}\text { Prepa- } \\
r \quad a \\
\text { maqui- } \\
\text { naria }\end{array}$ & $\begin{array}{l}P \text { e - } \\
\text { queñas } \\
\text { repara- } \\
\text { ciones }\end{array}$ & $\begin{array}{l}P r o- \\
\text { grama } \\
\text { maqui- } \\
\text { naria }\end{array}$ & $\begin{array}{l}\text { Busca } \\
\text { origen } \\
\text { defec- } \\
\text { tos }\end{array}$ & $\begin{array}{l}\text { S } 0 \text { o l } \\
\text { s } 0 \text { I } \\
\text { defec- } \\
\text { tos }\end{array}$ & $\begin{array}{l}s \circ 1 . \\
c \circ \quad n \\
\text { otros } \\
\text { defec- } \\
\text { tos }\end{array}$ & $\begin{array}{l}\text { Analiza } \\
\text { datos }\end{array}$ \\
\hline $\begin{array}{l}\text { A u - } \\
\text { tonomía } \\
\text { planifi- } \\
\text { cación }\end{array}$ & $\begin{array}{c}0,202 \\
\left(^{*}\right)\end{array}$ & $\begin{array}{c}0,276 \\
\left({ }^{* *}\right)\end{array}$ & 0,062 & $\begin{array}{c}0,316 \\
\left({ }^{* *}\right)\end{array}$ & $\begin{array}{c}0,264 \\
\left(^{*}\right)\end{array}$ & $\begin{array}{c}0,323 \\
\left({ }^{* *}\right)\end{array}$ & 0,154 & $\begin{array}{c}0,338 \\
\left({ }^{* *}\right)\end{array}$ & $\begin{array}{c}0,377 \\
\left({ }^{\star *}\right)\end{array}$ & $\begin{array}{c}0,392 \\
\left({ }^{* *}\right)\end{array}$ & 0,176 \\
\hline $\begin{array}{l}\text { A u - } \\
\text { tonomía } \\
\text { ritmos } \\
\text { trabajo }\end{array}$ & 0,128 & $\begin{array}{c}0,375 \\
\left({ }^{* *}\right)\end{array}$ & 0,128 & $\begin{array}{c}0,334 \\
\left({ }^{* *}\right)\end{array}$ & $\begin{array}{c}0,306 \\
\left({ }^{* *}\right)\end{array}$ & $\begin{array}{c}0,371 \\
\left({ }^{* *}\right)\end{array}$ & $0,230\left(^{* \star}\right)$ & $\begin{array}{c}0,356 \\
\left(^{* *}\right)\end{array}$ & $\begin{array}{c}0,400 \\
\left({ }^{* *}\right)\end{array}$ & $\begin{array}{c}0,296 \\
\left({ }^{* *}\right)\end{array}$ & $\begin{array}{c}0,289 \\
\left({ }^{* *}\right)\end{array}$ \\
\hline $\begin{array}{l}\text { A u - } \\
\text { tonomía } \\
\text { m é to - } \\
\text { dos }\end{array}$ & 0,011 & $\begin{array}{c}0,257 \\
\left(^{*}\right)\end{array}$ & 0,085 & $\begin{array}{c}0,210 \\
\left(^{*}\right)\end{array}$ & 0,163 & $\begin{array}{c}0,303 \\
\left({ }^{* *}\right)\end{array}$ & $0,265\left(^{(*}\right)$ & $\begin{array}{c}0,329 \\
\left({ }^{* *}\right)\end{array}$ & $\begin{array}{c}0,384 \\
\left({ }^{* *}\right)\end{array}$ & $\begin{array}{c}0,270 \\
\left({ }^{* *}\right)\end{array}$ & 0,134 \\
\hline
\end{tabular}

(†) $p<0,1 ;\left(^{*}\right) p<0,05 ;\left({ }^{* *}\right) p<0,01$

Tabla 11. Rho de Spearman entre el rediseño del trabajo y otras prácticas laborales (II)

\begin{tabular}{|l|c|c|c|}
\hline & Estandarización del trabajo & $\begin{array}{l}\text { Participación en elaborar tra- } \\
\text { bajo estándar }\end{array}$ & $\begin{array}{l}\text { Participación mejora del traba- } \\
\text { jo estándar }\end{array}$ \\
\hline $\begin{array}{l}\text { Autonomía en planificar y or- } \\
\text { ganizar el trabajo }\end{array}$ & $-0,22$ & $0,251\left(^{* *}\right)$ & $0,265\left(^{* *}\right)$ \\
\hline $\begin{array}{l}\text { Autonomía en establecer los rit- } \\
\text { mos de trabajo }\end{array}$ & 0,007 & $0,272\left(^{* *}\right)$ & $0,204\left({ }^{* *}\right)$ \\
\hline $\begin{array}{l}\text { Autonomía en establecer los } \\
\text { métodos del trabajo }\end{array}$ & $-0,165$ & $0,307\left({ }^{* *}\right)$ & 0,157 \\
\hline
\end{tabular}

El “Trabajo en equipo en el área de producción” solo presenta asociación significativa al $5 \%$ al emparejarlo con los ítems: "Planifica y organiza su trabajo" (rho=0,17, $\mathrm{p}$-valor $=0,030)$ y "Participa en la mejora continua", dando una intensidad medio-baja (rho $=0,314$, p-valor $=0,001$ ). Además, también al $10 \%$ con "Realiza mantenimiento básico" (rho=0,168; $\mathrm{p}$-valor=0,096) y "Par- ticipa en mejorar el trabajo estándarizado" (rho=0,186, $\mathrm{p}$-valor $=0,064)$.

A continuación se consideran simultáneamente varias prácticas laborales y se determinan las frecuencias relativas conjuntas que ofrecen las respectivas tablas de contingencia múltiples, 
Tabla 12. Utilización conjunta determinadas prácticas laborales. \% empresas

\begin{tabular}{|c|c|c|}
\hline Variable & $\geq 4$ & $\geq 7$ \\
\hline Autocontrol + Ordenar y limpiar & $80 \%$ & $49 \%$ \\
\hline Autocontrol + Ordenar y limpiar + Prepara la maquinaria & $68 \%$ & $27 \%$ \\
\hline Rotación entre puestos + Autocontrol + Ordenar y limpiar + Preparar la maquinaria & $53 \%$ & $12 \%$ \\
\hline Autocontrol + Ordenar y limpiar + Preparar la maquinaria + Participar en la mejora continua & $47 \%$ & $10 \%$ \\
\hline $\begin{array}{l}\text { Rotación entre puestos + Autocontrol + Ordenar y limpiar + Preparar la maquinaria + Participar en mejora } \\
\text { continua }\end{array}$ & $38 \%$ & $3 \%$ \\
\hline Autocontrol + Ordenar y limpiar + Part. mejora continua + Prepara maquinaria Mantenimiento básico & $34 \%$ & $8 \%$ \\
\hline $\begin{array}{l}\text { Rotación puestos +Autocontrol + Ordenar y limpiar + Preparar maquinaria + Part. mejora continua + Manto. } \\
\text { básico }\end{array}$ & $30 \%$ & $2 \%$ \\
\hline $\begin{array}{l}\text { Autocontrol + Ordenar y limpiar + Prepara maquinaria + Part. mejora continua + Pequeñas reparaciones + } \\
\text { Manto. básico }\end{array}$ & $28 \%$ & $6 \%$ \\
\hline $\begin{array}{l}\text { Autocontrol + Ordenar y limpiar + Part. mejora continua + Pequeñas reparaciones + Manto. básico + Preparar } \\
\text { la maquinaria + Buscar origen de los defectos }\end{array}$ & $7 \%$ & $0 \%$ \\
\hline $\begin{array}{l}\text { Autocontrol + Ordenar y limpiar + Participar en mejora continua + Pequeñas reparaciones + Manto. básico + } \\
\text { Preparar maquinaria + Buscar origen de los defectos +Solucionar solo los defectos }\end{array}$ & $4 \%$ & $0 \%$ \\
\hline $\begin{array}{l}\text { Autocontrol + Ordenar y limpiar + Part. mejora continua + Pequeñas reparaciones + Manto. básico + Preparar } \\
\text { maquinaria + Buscar origen defectos +Solucionar solo los defectos + Programar maquinaria }\end{array}$ & $1 \%$ & $0 \%$ \\
\hline
\end{tabular}

Sólo el $2 \%$ de las empresas incorporan simultáneamente en grado alto el "Trabajo en equipo en el área de producción", la "Rotación entre puestos", el "Autocontrol", "Ordena y limpia el puesto de trabajo", "Pequeñas reparaciones", "Prepara la maquinaria", "Mantenimiento básico" y "Participa en la mejora continua". Si además le añadimos "Buscar en origen los defectos", entonces ninguna empresa lo cumple.
Las frecuencias relativas conjuntas de considerar simultáneamente varias modalidades de remuneración variables (tabla 13), permiten comprobar que cuando se escogen al menos tres modalidades la proporción muestral es escasa y nula cuando se consideran todas las seleccionadas.

Tabla 13. Modalidades de remuneración variable para los operarios. Frecuencia relativa conjunta

\begin{tabular}{|l|c|}
\hline & Porcentaje \\
\hline Rendimiento / Productividad + Resultado de empresa & $7,0 \%$ \\
\hline Rendimiento / Productividad 'Incentivo cumplir objetivos del equipo & $6,0 \%$ \\
\hline Rendimiento / Productividad + Calidad conseguida & $5,0 \%$ \\
\hline Rendimiento / Productividad + Conocimientos/habilidades/competencias & $5,0 \%$ \\
\hline Incentivo por cumplir objetivos del equipo + Calidad conseguida & $4,0 \%$ \\
\hline
\end{tabular}




\begin{tabular}{|l|c|}
\hline Incentivo por cumplir objetivos del equipo + Resultado de empresa & $4,0 \%$ \\
\hline Incentivo por cumplir objetivos del equipo + Conocimientos/habilidades/competencias & $3,0 \%$ \\
\hline Resultado de empresa + Conocimientos/habilidades/competencias & $5,0 \%$ \\
\hline Rendimiento / Productividad + Calidad conseguida + Incentivo cumplir objetivos del equipo & $3,0 \%$ \\
\hline $\begin{array}{l}\text { Incentivo por cumplir objetivos del equipo + Resultado de empresa + Conocimientos/hab- } \\
\text { ilidades/competencias }\end{array}$ & $1.0 \%$ \\
\hline $\begin{array}{l}\text { Rendimiento / Productividad + Incentivo por cumplir objetivos del equipo + Resultado de } \\
\text { empresa }\end{array}$ & $1,0 \%$ \\
\hline $\begin{array}{l}\text { Rendimiento / Productividad + Incentivo por cumplir objetivos del equipo + Calidad obteni- } \\
\text { da + Resultado de empresa }\end{array}$ & \\
\hline $\begin{array}{l}\text { Rendimiento / Productividad + Incentivo cumplir objetivos del equipo + Calidad obtenida + } \\
\text { Rdo. empresa + Rdo. planta }\end{array}$ & $1.0 \%$ \\
\hline $\begin{array}{l}\text { Rendimiento / Productividad + Incentivo por cumplir objetivos del equipo + Calidad obteni- } \\
\text { da + Resultado de empresa + Conocimientos/ habilidades/capacidades }\end{array}$ & $1,0 \%$ \\
\hline
\end{tabular}

Las modalidades del salario variable se han recogido como atributos dicotómicos (categorías: Si/No), y al contrastar como hipótesis nula la independencia entre pares de las mismas, se rechaza al considerar el par "Incentivos por cumplir objetivos del equipo" y "Calidad conseguida" (estadístico exacto de Fisher con p-valor=0,017), cuya intensidad de asociación es medio-alta $(Q$ de Yule $=0,74)$, así como entre las modalidades: "Resultado de la empresa" y "Conocimientos/Capacidades/Habilidades del trabajador" (estadístico exacto de Fisher con $p$-valor $=0,034)$, con intensidad media $(Q$ de Yule=0,62).

La tabla 14 presenta los resultados de las tablas de contingencia al cruzar las modalidades de salario vari- able y las prácticas laborales categorizadas (Bajo=0-3, Medio=4-6 y Alto: $\geq 7$ ) en las que se rechaza la hipótesis nula de independencia, mediante el contraste chi cuadrado, y la intensidad de asociación con el estadístico $\mathrm{V}$ de Cramer, cuyos valores suelen ser medio-bajos, y para ningún par de ítems es elevada. Los dos mayores valores del estadístico corresponden a la modalidad "Salario variable por conocimientos/capacidades/habilidades" con el ítem "Realiza mantenimiento básico" (V-Cramer = 0,377), y con el ítem "Participa en la mejora continua" (V-Cramer $=0,341)$. La modalidad "salario variable por conocimientos/ capacidades/habilidades" se asocia con la mayoría de ítems considerados en la flexibilidad funcional.

Tabla 14. Asociación entre prácticas laborales y modalidades de salario variable

\begin{tabular}{|c|c|c|c|}
\hline & Chi cuadrado & p-valor & V-Cramer \\
\hline Autonomía en planificar y organizar el trabajo * Resultado planta & 7,216 & 0,027 & 0,269 \\
\hline Autonomía en planificar y organizar el trabajo * Resultado de empresa & 7,617 & 0,022 & 0,276 \\
\hline Autonomía en planificar y organizar el trabajo * Conocimientos/habilidades/capacidades operario & 7,081 & 0,029 & 0,266 \\
\hline Autonomía en ritmos de trabajo * Calidad obtenida & 5,047 & 0,080 & 0,225 \\
\hline Autonomía en ritmos de trabajo * Incentivos por cumplir objetivos del equipo & 6,064 & 0,048 & 0,246 \\
\hline
\end{tabular}




\begin{tabular}{|c|c|c|c|}
\hline Autonomía en escoger métodos de trabajo * Conocimientos/habilidades/capacidades operarios & 5,085 & 0,079 & 0,226 \\
\hline Participación en la mejora continua * Rendimiento/Productividad & 5,303 & 0,071 & 0,230 \\
\hline Participación en la mejora continua * Incentivos por cumplir objetivos del equipo & 6,396 & 0,041 & 0,253 \\
\hline Participación en la mejora continua * Conocimientos/habilidades/capacidades operarios & 11,662 & 0,003 & 0,341 \\
\hline Ordenar y limpiar puesto trabajo * Calidad conseguida & 7,719 & 0,021 & 0,278 \\
\hline Pequeñas reparaciones * Calidad conseguida & 5,236 & 0,073 & 0,229 \\
\hline Pequeñas reparaciones * Incentivos por cumplir objetivos del equipo & 5,932 & 0,051 & 0,244 \\
\hline Pequeñas reparaciones * Conocimientos/habilidades/capacidades operarios & 7,555 & 0,023 & 0,275 \\
\hline Mantenimiento básico * Incentivos por cumplir objetivos del equipo & 5,712 & 0,059 & 0,239 \\
\hline Mantenimiento básico * Conocimientos/habilidades/capacidades operarios & 14,184 & 0,001 & 0,377 \\
\hline Busca origen defectos * Conocimientos/habilidades/capacidades operarios & 5,75 & 0,056 & 0,240 \\
\hline Soluciona solo los defectos * Incentivos por cumplir objetivos del equipo & 6,374 & 0,041 & 0,252 \\
\hline Soluciona con otros los defectos * Calidad conseguida & 5,302 & 0,071 & 0,230 \\
\hline Rotación entre puestos * Resultado de la empresa & 4,834 & 0,089 & 0,220 \\
\hline Equipos de mejora voluntarios * Conocimientos/habilidades/capacidades operarios & 5,893 & 0,053 & 0,243 \\
\hline
\end{tabular}

\subsubsection{Contrastes de igualdad en la medida de posición central según la variable de agrupación seleccionada}

Para los ítems "Ritmos de trabajo", "Nivel de stress" y "Absentismo", se busca en cada caso individual si hay diferencias significativas según cada tipo de flexibilidad funcional seleccionado, considerando las categorías: baja (0-3), medio (4-6) y alto ( $\geq 7)$. Por ello se plantea la hipótesis nula:

$\rightarrow$ H10: Igualdad en la medida de posición central del ítem (Ritmos de trabajo/Nivel de Estrés/ Absentismo), segmentando según el grado del tipo de flexibilidad funcional

Aplicando el test de Kruskal y Wallis, la variable "Ritmos de trabajo" presenta diferencias significativas al $10 \%$ al segmentar por el ítem "Autonomía del operario para establecer sus ritmos de trabajo" (chi cuadrado=5,906; p-valor $=0,052$ ) con rangos promedios: bajo $=56,05$; medio=48,50; alto=37,18. Por tanto, la relación es inversa entre ambos ítems. Asimismo, también es significativa al segmentar según la "Autonomía del operario en es- tablecer sus métodos de trabajo" (chi cuadrado=4,808; $\mathrm{p}$-valor $=0,090$ ) con rangos promedios asociados a las categorías: bajo=54,94; medio=42,64; alto=40,50. Estos resultados permiten afirmar que los ritmos de trabajo son superiores cuando es reducida la autonomía del operario para establecer sus ritmos y métodos de trabajo.

De la aplicación del test de Kruskal y Wallis se verifica también que hay diferencias significativas al $10 \%$ en los ritmos de trabajo (chi cuadrado=6;919 y p-valor $=0,085$ ) al segmentar por el grado de implantación del "Trabajo en equipo en el área de producción", obteniéndose los siguientes rangos promedios para las modalidades: nulo $=46,50$; bajo $=40,96$; medio $=61,24$; alto $=60.34$. Por tanto, el ritmo de trabajo es mayor al aplicarse el trabajo en equipo en producción al menos con grado medio. Además, hay evidencia empírica suficiente al 5\% para rechazar la medida de posición central de los ritmos de trabajo y del nivel de absentismo, al segmentar según el ítem "Rotación entre puestos", siendo inferior el nivel de ambos ítems cuando la rotación de puestos es baja, como reflejan los siguientes valores del rango promedio (ver tabla 15). 
Tabla 15. Contraste de Kruskal y Wallis de los ritmos de trabajo y del absentismo, segmentando según la rotación entre puestos de trabajo

\begin{tabular}{|l|l|l|l|l|l|}
\hline & $\begin{array}{l}\text { C h i } \\
\text { cuad - } \\
\text { rado }\end{array}$ & $\begin{array}{l}\text { p-val- } \\
\text { or }\end{array}$ & $\begin{array}{l}\text { Rango } \\
\text { p r o - } \\
\text { medio } \\
\text { (Bajo) }\end{array}$ & $\begin{array}{l}\text { Rango } \\
\text { p r o - } \\
\text { medio } \\
\text { ( M e - } \\
\text { dio) }\end{array}$ & $\begin{array}{l}\text { Rango } \\
\text { p r - } \\
\text { medio } \\
\text { (Alto) }\end{array}$ \\
\hline $\begin{array}{l}\text { Rit m o s } \\
\text { de traba- } \\
\text { jo }\end{array}$ & 8,388 & 0,015 & 39,90 & 46,16 & 59,95 \\
\hline $\begin{array}{l}\text { Absentis- } \\
\text { mo }\end{array}$ & 6,110 & 0,047 & 36,81 & 55,09 & 53,26 \\
\hline
\end{tabular}

En cuanto al "Nivel de estrés", los resultados del test de Kruskal y Wallis verifican diferencias significativas al $5 \%$ cuando la variable de agrupación es la "Participación del operario en la mejora continua" (chi cuadrado=7,126; $p$-valor $=0,028$ ) con los siguientes rangos promedios según el grado de participación: bajo $=58,37$; medio $=48,65$; alto $=37,98$. Por tanto, dándose una relación inversa entre ambos ítems.

Si alternativamente se considera el "porcentaje de salario variable sobre el salario total" y como variable de agrupación cada uno de los ítems de flexibilidad funcional, entonces se establece como hipótesis nula:

$\rightarrow$ H2o: Igualdad en la medida de posición central del porcentaje del salario variable sobre el salario total, segmentando según el grado del ítem de flexibilidad funcional seleccionado

La aplicación del test de Kruskal y Wallis permite afirmar que hay diferencias significativas al $10 \%$ si la variable de agrupación es la "Autonomía del operario en organizar y planificar su trabajo" (chi cuadrado $=5,332$; p-valor $=0,07)$, con rangos promedios crecientes (bajo=44,96; medio=54,50; alto=62,60). Asimismo, al escoger el "Operario ordena y limpia su puesto de trabajo" (chi cuadrado $=5,54$; p-valor $=0,063$ ), se obtienen tambien rangos promedios crecientes (bajo $=36,75$; medio=38,78; alto=54,31). Por tanto, la relación es directa entre el salario variable y la aplicación de ambos ítems.

A continuación, alternativamente se consideran los ratios de productividad del trabajo, margen de explotación y gastos de personal/ingresos de explotación y se mantienen las variables de agrupación para aplicar el test de Kruskal y Wallis.

Para el ratio de productividad laboral ("Valor añadido/ Personal"), se establece la siguiente hipótesis nula propuesta:

$\rightarrow$ H3o: Igualdad en la medida de posición central de la productividad del trabajo, segmentando según el grado del ítem de flexibilidad funcional seleccionado

Se concluye que no hay evidencia empírica suficiente para rechazar la citada hipótesis nula al segmentar por las diferentes modalidades de flexibilidad funcional, considerando las categorías: bajo (0-3), medio (4-6), alto $(\geq 7)$.

Si se considera el margen de explotación, dado por el ratio Resultado de explotación/ Ingresos de explotación, entonces la hipótesis nula es:

$\rightarrow$ H4o: Igualdad en la medida de posición central del margen de explotación segmentando según el grado del ítem de flexibilidad funcional seleccionado

Se obtienen diferencias significativas en los ítems de la tabla 16, cuyos rangos promedios muestran que el margen bruto de explotación es inferior cuando la intensidad del ítem es baja.

Tabla 16. Contraste de Kruskal y Wallis del ratio Resultado de explotación / Ingresos explotación segmentando según determinadas prácticas laborales

\begin{tabular}{|l|c|c|c|c|c|}
\hline & Chi cuadrado & p-valor & $\begin{array}{c}\text { Rango promedio } \\
\text { (Bajo) }\end{array}$ & $\begin{array}{c}\text { Rango promedio } \\
\text { (Medio) }\end{array}$ & $\begin{array}{c}\text { Rango promedio } \\
\text { (Alto) }\end{array}$ \\
\hline $\begin{array}{l}\text { Autonomía operario en organizar y } \\
\text { planificar el trabajo }\end{array}$ & 5,515 & 0,063 & 43,84 & 56,68 & 58,80 \\
\hline $\begin{array}{l}\text { Autonomía operario en establecer } \\
\text { ritmos de trabajo }\end{array}$ & 13,53 & 0,001 & 39,80 & 62,58 & 57,62 \\
\hline Autocontrol & 6,179 & 0,046 & 36,11 & 48,08 & 56,19 \\
\hline
\end{tabular}




\begin{tabular}{|l|c|c|c|c|c|}
\hline $\begin{array}{l}\text { Operario ordena y limpia puesto de } \\
\text { trabajo }\end{array}$ & 4,619 & 0,099 & 31,00 & 40,95 & 53,26 \\
\hline $\begin{array}{l}\text { Operario realiza mantenimiento } \\
\text { básico }\end{array}$ & 8,325 & 0,016 & 42,44 & 51,73 & 62,72 \\
\hline
\end{tabular}

$\mathrm{Si}$ el ratio a considerar es "Gastos de personal//ngresos de explotación", la hipótesis nula es:

$\rightarrow$ H5o: Igualdad en la medida de posición central del ratio gastos de personal sobre ingresos de e $x$ plotación, segmentando según el grado del ítem de flexibilidad funcional seleccionado

Se comprueba que sólo hay diferencias significativas al $10 \%$ en la medida de posición central del ratio si la variable de agrupación es la "Participación del operario en la mejora continua" (Chi cuadrado $=4,657$; $\mathrm{p}$-valor $=0,097)$, con valores de los rangos promedios decrecientes (bajo=56,03; medio $=49,41$; alto=39,1).

Si para los tres ratios se considera alternativamente como variable de agrupación la modalidad de salario variable seleccionada, las hipótesis nulas a contrastar son:

$\rightarrow$ H6o: Igualdad en la medida de posición central de los ratios: productividad laboral, margen de explotación y gastos de personal sobre ingresos de explotación segmentando según el la modalidad de salario variable seleccionada

El ratio de productividad laboral sólo ofrece diferencias significativas al segmentar por el salario variable según el resultado de la planta $(U$ de Mann Whitney $=48$; $p$-valor $=0,045$ y rangos promedios: $\mathrm{No}=48,98$; $\mathrm{Si}=82,67)$.

Para el ratio "Gastos de personal/Ingresos de explotación" hay diferencias significativas al $10 \%$ cuando la variable de agrupación es el "salario variable según el resultado de la planta" (U de Mann Whitney=50; $p$-valor 0,55$)$ con rango promedio superior al no aplicarse $(\mathrm{No}=50 ; \mathrm{Si}=18,67)$ y también al $5 \%$ si se considera como variable de agrupación la modalidad "salario variable según los conocimientos/habilidades/ capacidades del operario" (U de Mann Whitney $=377$; $\mathrm{p}$-valor $=0,029)$ y rango promedio superior al disponerlo $(\mathrm{No}=37,33 ; \mathrm{Si}=65,57)$. Por tanto, la disponibilidad de ambas modalidades parece incidir en que sea superior el peso de los gastos de personal sobre los ingresos de explotación.

Para el ratio "Margen de explotación" no se verifican diferencias significativas para ninguna modalidad escogida de salario variable.

\subsubsection{Análisis Homals y Cluster}

Se aplica el Homals (Homogeneity analysis by means of alternating least squares) para obtener relaciones entre más de dos variables, buscando un número reducido de dimensiones que permitan retener una parte significativa de la variación total. Se escogen los ítems valorados según las modalidades: bajo $[\leq 3]$, medio $[4,6$ ] y alto [ $\geq 7]$.

De su aplicación se obtienen las dos primeras dimensiones, que suponen el $45,8 \%$ de varianza retenida. La tabla 17 refleja las medidas de discriminación de las variables en las dimensiones especificadas. La primera dimensión viene explicada en orden a su contribución principalmente por las variables señaladas en negrita, que recogen diversas tareas o funciones delegadas en el operario, destacando buscar el origen de los defectos y posterior solución individual o en grupo, junto a programar la maquinaria. Le siguen que analiza datos y hace el mantenimiento básico. En cambio, la segunda dimensión viene discriminada principalmente por incorporar equipos y la participación del operario en la mejora continua. 
Tabla 17. Medidas de discriminación

\begin{tabular}{|c|c|c|c|}
\hline & DIM 1 & DIM 2 & Media \\
\hline El operario busca el origen defectos & 0,619 & 0,083 & 0,351 \\
\hline El operario soluciona solo los defectos & 0,602 & 0,006 & 0,304 \\
\hline El operario programa la maquinaria & 0,515 & 0,071 & 0,293 \\
\hline El operario soluciona con otros los defectos & 0,462 & 0,036 & 0,249 \\
\hline El operario analizar datos & 0,386 & 0,009 & 0,198 \\
\hline El operario realiza mantenimiento básico & 0,343 & 0,053 & 0,198 \\
\hline Autonomía en la planificación y organización del trabajo & 0,260 & 0,064 & 0,162 \\
\hline Autonomía en escoger métodos de trabajo & 0,236 & 0,072 & 0,154 \\
\hline Trabajo en equipo en área de producción & 0,007 & 0,535 & 0,271 \\
\hline Equipos de mejora voluntarios & 0,057 & 0,448 & 0,253 \\
\hline Participa el operario en la mejora continua & 0,065 & 0,427 & 0,246 \\
\hline Equipos diferentes departamentos para solucionar problemas & 0,030 & 0,291 & 0,161 \\
\hline Rotación entre puestos de trabajo & 0,012 & 0,143 & 0,078 \\
\hline Porcentaje de varianza & 29,663 & 16,149 & 22,906 \\
\hline
\end{tabular}

\section{Análisis Cluster}

Se aplica el análisis cluster sobre los puntos objetos obtenidos del análisis Homals, seleccionando el procedimiento de Ward y como medida la distancia euclídea al cuadrado. El dendograma permite observar 10 grupos, según el grado de incorporación de los ítems recogidos en la tabla de las medidas de discriminación. A continuación se comentan sus características principales.

- Grupo 1 (17\%): Carecen o están escasamente implantados los ítems de la tabla 16. Las empresas disponen una organización de tipo taylorista-fordista

- Grupo 2 (13\%). La mayoría de los ítems ofrecen valores nulos o reducidos, si bien se valora en grado medio que el operario "Busca el origen de los defectos" y/o "Soluciona con otros los defectos". Se puede considerar también básicamente de organización taylorista-fordista.

- Grupo 3 (16\%). Generalmente los operarios solucionan los defectos de forma individual o en grupo y participan en la mejora continua, en grado medio. Suelen encargarse del mantenimiento básico, al menos en grado medio-bajo. La mayoría incorpora el trabajo en equipo y tienen equipos de diferentes departamentos para solucionar problemas, al menos en grado medio, pero frecuentemente se carece de los equipos de mejora voluntarios o se utilizan débilmente. La implantación del resto de ítems de la tabla 16 es nula o reducida.

- Grupo 4 (3\%). El operario participa en la mejora continua en grado medio, y respecto el trabajo en equipo, los equipos de mejora voluntarios y los equipos de diferentes departamentos para solucionar problemas, dos de tales ítems están implantados en grado alto. El resto de ítems se implantan de forma escasa o bien es nula.

- Grupo 5 (9\%). Generalmente el operario no busca el origen de los defectos o lo efectúa en grado bajo. En cambio, en todas, el operario soluciona sólo o con otros los defectos, al menos en grado medio. El operario también suele encargarse del mantenimiento básico al menos en grado medio. El trabajo en equipo está muy implantado, y la mayoría utiliza intensamente los 
equipos de mejora voluntarios y/o equipos interdepartamentales para solucionar problemas.

- Grupo 6 (6\%). Todas las empresas incorporan de forma conjunta al menos en grado medio los ítems: el operario busca el origen de los defectos, soluciona solo y con otros los defectos, programa su maquinaria, analiza datos, y hace mantenimiento básico. También al menos en grado medio la autonomía del operario para planificar y organizar el trabajo, así como en la autonomía en métodos de trabajo, salvo una empresa. En cambio, la mayoría no tienen implantado o escasamente el trabajo en equipo, los equipos de mejora voluntarios y los equipos de diferentes departamentos para solucionar problemas.

- Grupo 7 (15\%). El operario busca intensamente el origen de los defectos, soluciona solo o con otros los defectos y programa la maquinaria. En la mayoría el operario realiza el mantenimiento básico y analiza datos, al menos en grado medio, así como seleccionar los métodos de trabajo en grado medio. En cambio, se carece o es débil el trabajo en equipo, los equipos de mejora voluntarios y los equipos interdepartamentales para solucionar problemas.

- Grupo 8 (8\%). En la mayoría el operario intensamente busca el origen de los defectos, soluciona solo los defectos, soluciona con otros los defectos, programa la maquinaria, analiza datos, se encarga del mantenimiento básico y participa en la mejora continua. Asimismo, la autonomía y planificación del trabajo es al menos de grado medio y mayoritariamente elevada. Además generalmente el operario dispone de autonomía para escoger los métodos de trabajo, se tienen equipos de diferentes departamentos para solucionar problemas y equipos de mejora voluntarios, al menos en grado medio. Por tanto, ofrece una destacada flexibilidad funcional

- Grupo 9 (4\%). Los operarios solucionan solos o en grupo los defectos en grado medio e intensamente realizan el mantenimiento básico. En la mayoría planifica y organiza el trabajo, participa en la mejora continua y analiza datos al menos en grado medio, sin embargo, escasamente busca los orígenes de los defectos. Asimismo disponen de equipos de mejora voluntarios y equipos de diferentes departamentos para solucionar problemas al menos en grado medio-bajo. En cambio, el trabajo en equipo es reducido o nulo.

- Grupo 10 (9\%). El operario soluciona sólo o con otros los defectos en grado alto, programa la maquinaria intensamente (salto salvo en dos empresas), busca el origen de los defectos y analizan datos, al menos en grado medio, pero generalmente realiza poco man- tenimiento. La mayoría carece del trabajo en equipo y de los equipos de mejora, aunque suele disponer de equipos de diferentes departamentos para solucionar problemas, con intensidad media.

A continuación se aplica el test de Kruskal y Wallis considerando como hipótesis nula la igualdad en la medida de posición central de los ítems: productividad laboral, margen explotación, ritmos de trabajo, nivel de stress y grado de absentismo, segmentando según los grupos obtenidos en el análisis cluster. La conclusión es que no hay evidencia empírica para rechazar tales hipótesis nulas (ver tabla 18).

Tabla 18. Contraste de Kruskal y Wallis sobre la productividad laboral, los ritmos de trabajo, el nivel de stress y el absentismo, segmentando según los grupos del cluster

\begin{tabular}{|l|l|l|l|l|l|}
\hline & $\begin{array}{l}\text { V.A.I } \\
\text { Plantil- } \\
\text { la }\end{array}$ & $\begin{array}{l}\text { R d o. } \\
\text { exp..I } \\
\text { In g r. } \\
\text { exp }\end{array}$ & $\begin{array}{l}\text { Ritmos } \\
\text { de tra- } \\
\text { bajo. }\end{array}$ & $\begin{array}{l}\text { N i v - } \\
\text { el de } \\
\text { stress }\end{array}$ & $\begin{array}{l}\text { A b - } \\
\text { s e n t - } \\
\text { ismo }\end{array}$ \\
\hline $\begin{array}{l}\text { C h i } \\
\text { cua d- } \\
\text { rado }\end{array}$ & 11,946 & 9,570 & 11,306 & 2,320 & 10,498 \\
\hline p-valor & 0,216 & 0,386 & 0,255 & 0,985 & 0,312 \\
\hline
\end{tabular}

\section{Conclusiones, discusión, limi- taciones y líneas de futuro}

La mayoría de empresas delegan bastante en sus operarios determinadas tareas indirectas, destacando el orden y limpieza de su puesto de trabajo (base de las $5 S$ ), el autocontrol, y preparar la maquinaria. En cambio, es reducido el rediseño del trabajo, medido con la autonomía en planificar y organizar el trabajo, establecer los ritmos y métodos de su trabajo, lo que puede afectarle negativamente en la motivación y satisfacción del trabajo. Además, el operario es quien mejor conoce la parte del proceso productivo donde desarrolla su trabajo, por lo que debería participar más en la mejora continua, ayudando a optimizar los procesos y buscar eficiencias, para mejorar la productividad y reducir los costes. El hecho de que en la mayoría suele efectuar escasamente el mantenimiento básico imposibilita o dificulta la implantación del TPM, sistema que más empresas deberían incorporar por las ventajas que ofrece. Asimismo, el operario debería participar más en la determinación de los estándares que afectan a su trabajo y su posterior mejora, para ajustarse a sus necesidades y capacidades, aumentando ello su motivación en el trabajo. 
La rotación entre puestos está bastante implantada, pero se asocia a mayores ritmos de trabajo y nivel de absentismo, lo que afecta negativamente a la calidad de la vida laboral. Debería mejorarse el diseño de la rotación entre puestos dando mayor relevancia a la ergonomía en los puestos de trabajo. Los ritmos de trabajo también son superiores cuando la autonomía del operario para establecer sus ritmos y métodos de trabajo es reducida.

El trabajo en equipo en el área de producción no llega a aplicarse en la mitad de empresas, tendiendo al modelo de la producción ajustada, con las ventajas que ofrece para eliminar despilfarros, mejorar la calidad, el servicio y la innovación, si bien también se verifica que su mayor implantación aumenta los ritmos de trabajo, lo que en un futuro puede generar problemas de salud en los operarios de mayor edad que trabaja en los mismos y se incremente su nivel de absentismo. Por otra parte, la elevada proporción de personal con bajo nivel de estudios en la mayoría de empresas dificulta que el operario pueda asumir en los equipos un superior enriquecimiento vertical de su trabajo y dificulte pueda delegarse actividades indirectas de mayor complejidad (p.e. de mantenimiento).

El peso del salario variable sobre el salario total suele ser reducido, no superando la mayoría el 18\%, y se aplica más en las empresas donde el operario tiene mayor autonomía en planificar y organizar el trabajo, así como en escoger sus métodos de trabajo. Por modalidades de salario variable, los incentivos de rendimiento/productividad son los más utilizados (aunque sin llegar a la tercera parte de las empresas), seguido de los incentivos de equipos, pero carecen de asociación con el resto de prácticas laborales. Sin embargo, la modalidad de salario variable por "conocimientos/ habilidades/capacidad" ofrecen mayor asociación con diversas medidas de flexibilidad laboral. Por tanto, la aplicación de una forma bastante aislada de tales incentivos respecto a otras prácticas laborales consideradas puede provocar que no se obtengan los resultados deseados, que podrían conseguirse con una mayor complementariedad entre los mismos, para obtener sinergias. Por ello, sería conveniente aplicar más los incentivos de equipo al trabajar en equipo para motivar a sus integrantes y también elementos salariales según el rendimiento o productividad, que incentiven y motiven a aquellos trabajadores que individualmente ofrecen mayor output. Asimismo, hacer más partícipes a los trabajadores de los beneficios de planta o empresa por las ventajas que suponen.

La utilización conjunta de la mayoría de prácticas laborales de tareas indirectas consideradas en grado alto es muy escasa y en su totalidad nula. Asimismo, la aplicación conjunta de las modalidades de salario variable consideradas es reducida y en su totalidad también nula. Por tanto, de las sinergias y complementariedades que proporcionan su incorporación conjunta pueden beneficiarse a pocas empresas.

En el análisis Homals la primera dimensión recoge la mayoría de tareas indirectas delegadas al operario, que están más relacionadas entre sí: buscar el origen de los defectos, solucionar los defectos, programar la maquinara, analizar datos y hacer el mantenimiento básico, además de la autonomía del operario para organizar y planificar su trabajo, así como de escoger los métodos de trabajo. En cambio, la segunda dimensión discrimina por trabajar en equipo en la línea de producción y fuera de la misma, junto a la participación del trabajador en la mejora continua, facilitando conjuntamente la mejora continua y la solución de problemas. El posterior análisis cluster permite verificar la hibridación en la mayoría de empresas por la aplicación de tales ítems. Esa variedad puede venir dada por diversas circunstancias, tales como el capital humano disponible, la incorporación de sistemas de automatización avanzados, las exigencias salariales de los sindicatos por asumir el operario más responsabilidades y la cultura corporativa implantada.

No se observan diferencias significativas en la productividad del trabajo al segmentar según el grado de aplicación individual de las modalidades de flexibilidad funcional, ni por los grupos obtenidos en el análisis cluster. En cambio, el margen operativo si ofrece diferencias significativas según el grado de aplicación de ciertas delegaciones en el trabajador (autonomía en planificar y organizar el trabajo, participar en la mejora continua, realizar pequeñas reparaciones y el mantenimiento básico, buscar el origen de los defectos y solucionar solo los defectos), pero sin embargo, no se obtienen al segmentar por los grupos del cluster, lo que puede ser consecuencia de la falta de sinergias por aplicarse más de forma aislada o escasamente de forma conjunta.

En cuanto a las limitaciones del estudio, debe considerarse que sólo recoge datos de las empresas medianas y grandes, no siendo extrapolable a las pequeñas empresas. Los resultados pueden variar respecto de otras regiones y países (la composición de los subsectores puede variar, así como el grado de presencia de empresas de capital extranjero, nacional y tamaño empresarial), así como respecto de otros sectores.

Los datos son de corte transversal, lo que no permite ver una evolución, y en su mayoría son ordinales. Algunos ítems podrían haberse planteado alternativamente como variables cuantitativas (p.e. porcentaje de 
operarios que trabajan en equipo, rotan entre puestos, participan en la mejora continua, etc.), pero entonces es previsible una sensible menor tasa de respuesta, lo que haría menos representativa a la muestra, al requerir buscar o elaborar tales datos por parte de responsables de la empresa y además generarle una mayor pérdida de confidencialidad, lo que suele implicar desde la Dirección una mayor aversión a facilitarlos, especialmente en épocas de crisis. Por ello, se ha escogido para la mayoría de variables alternativamente las percepciones de los presidentes de los Comités de Empresa, que consideramos tienen adecuado conocimiento de la mayoría de ítems requeridos y cuando lo desconozcan, entonces buscar obtener la información por parte de la Dirección. Sin embargo, debe considerarse que las percepciones de los presidentes de los comités de empresa en algunos ítems pueden variar de las que tengan los responsables de Recursos Humanos (porque en algunos casos sus respuestas se pueden ajustar menos a la veracidad, para dar una mejor imagen de la empresa).

Como línea de futuro con la presente base de datos se puede analizar si hay diferencias significativas según la nacionalidad, tamaño empresarial y antigüedad de la empresa. El cuestionario recoge una parte de los ítems de flexibilidad funcional y podrían ampliarse con nuevas variables para efectuar un nuevo estudio, en cuyo cuestionario incorporar nuevos ítems como los tipos de estrategia (p.e. las que ofrecen Porter o Miles y Show), que puede ser una variable moderadora entre el sistema de salarial, la flexibilidad funcional y el perfomance de la organización. Se podría también incorporarla como variable de control, mediante dummies, si se quiere elaborar un modelo de regresión para explicar variables como la productividad, la rentabilidad empresarial o el gasto de personal /Plantilla.

Sería conveniente disponer también de información sobre la tecnología de proceso que las empresas incorporan, como qué sistemas de producción flexibles incorpora u otros sistemas de automatización avanzados han implantado, que junto al grado de formación dado al trabajador pueden tener incidencia en la organización del trabajo (facilitando incorporar ciertas prácticas laborales asociadas a la producción ajustada) y en la productividad que obtiene la empresa. Asimismo, incorporar el ítem subsectores como variable de control, y también como variable explicativa la complejidad del producto que fabrica la empresa (según el grado de valor añadido incorpore: alto, medio o bajo) y la percepción sobre el dinamismo del entorno. Sería asimismo interesante conseguir el grado de sindicación en la empresa, el nivel de participación, conocer la posible existencia de acuerdos y/o apoyo de los sindicatos en la adopción e implantación de las medidas de flexibili- dad funcional y salarial. Otro aspecto interesante sería saber si la empresa realiza innovaciones de productos, innovaciones de procesos, y el valor del ratio gastos de I+D/Ventas, para verificar su posible relación con la flexibilidad funcional y salarial.

A partir de tales variables se puede intentar de crear un modelo logit para explicar la presencia o ausencia de incorporar determinadas prácticas laborales (p.e. el trabajo en equipo en el área de producción). Alternativamente se podría buscar establecer un multinomial ordenado, dado que se tiene una valoración de escala de 0 a 10 en la mayoría de variables. Por otra parte, tales variables también se pueden utilizar como variables explicativas de la productividad y rentabilidad empresarial. Además, se podría incorporar en el nuevo cuestionario otros indicadores de la empresa, tales como porcentajes de entregas a tiempo, porcentajes de defectos, pues pueden ser variables dependientes de las medidas de flexibilidad funcional implantadas por las empresas. Otro indicador interesante sería el valorar la satisfacción del trabajador, medida con una escala de 0 a 10, e implantar un modelo de ecuaciones estructurales en el que esa variable satisfacción del trabajador fuese la variable resultado, considerando en el mismo como variables explicativas los ítems de flexibilidad funcional y salarial, junto al resto de variables mediadoras y de control comentadas anteriormente a obtener en una nueva encuesta, incorporándolas en el cuestionario.

\section{Bibliografía}

ADLER, P.S. y COLE, R. (1995). "Designed for learning: A tale of two auto plants,". En Ake Sandberg (ed.): Enriching Production Volvo's Uddevalla plant as an alternative to lean production, Aldershot: Avebury, Ashagaste Publishing Limited, pp. 157-178.

ARYA, A., Y MITTENDORF, B. (2004). "Using Job Rotation to Extract Employee Information," The Journal of Law, Economics, \& Organization, 20 (2), pp. 400414

ALCHIAN, A. y DEMSETZ, H. (1972). Production, information, costs and economic organization", American Economic Review, 62 (5), pp. 777-795

APPELBAUM, E.y BATT, R. (1994). The new American workplace. New York: ILR Press, Ithaca

APPELBAUM, E.y BATT, R. (1995). "Worker Participation in Diverse Settings: Does the Form affect the Outcome, and if so, who benefits?" British Journal of Industrial Relations, 33 (3), pp. 354-378 
APPELBAUM, E., BAYLEY, T., BERG, P. y KALLEBER, A. (2000). Manufacturing adcantage. Why high perfomance work systems pay off, Londres: ILR Press, Cornell University Press, Ithaca

ARUNDEL, A.; EDWARD, E., LUDVALL, B.A., VALEYRE, A. (2007). "How Europe's economies learn: a comparison of work organization and innovation mode for the EU-15", Industrial and Corporate Change, 16, (6), pp. 1175-1210

ARVANITIS, S. (2005). "Modes of labor flexibility at firm level: Are there any implications for performance and innovation?". Evidence for the Swiss economy", Industrial and Corporate Change, 14 (6), pp. 993-1016

ASPLUND, R. y OKSANEN, J. (2003). Functional flexibility strategies: evidence from companies in five small european economies, Research Institute of the Finnish Economy Discussion, ETLA, Discussion Papers, $n^{\circ}$ 874, Helsinki.

ATKINSON, J. (1984). "Manpower strategies for flexible organizations", Personnel Management, 16 (8), pp. $32-35$

BARTH, E., BRATSBERG, B, y HAEGELAND,T. (2008). "Who pay for perfomance?", International Journal of Manpower, 29 (1), pp. 8-29

BATT, R, y APPELBAUM, E. (1995). "Worker participation in diverse settings: does the form afffect the outcome and it so, who benefits". British Journal of Industrial Relations, 33 (3), pp. 353-378

BAUER, T.K. (2004). 'High Performance Workplace practices and job satisfaction: evidence from Europe', IZA Discussion Papers, Nº 1265

BAYO, A. y MERINO, J. (2002). "Las prácticas de recursos humanos de alto compromiso: un estudio de los factores que influyen sobre su adopción en la industria española", Cuadernos de Economía y Dirección de la Empresa, 12, pp. 227-246

BAYO-MORIONES, A. y HUERTA ARRIBAS, A. (2002a). "The adoption of production incentives in Spain", British Journal of Industrial Relations, 40 (4), pp. $709-724$

BAYO-MORIONES, A. y HUERTA ARRIBAS, A. (2002b). "Organisational incentive plans in Spanish manufacturing industry", Personnel Review, 31 (2), pp. $128-142$
BAYO-MORIONES, A. y HUERTA ARRIBAS, A (2003). El pago de incentivos en la empresa industrial española, Fundación BBVA, Bilbao.

BAYO-MORIONES, A., BELLO-PINTADO, A. y MERINO-SÍAZA DE CERIO, J. (2010). "5S use in manufacturing plants contextual factors and impact on operating perfomance", International Journal of Quality \& Reliability Management, 27 (2), pp. 217-230

BEARDWELL, I. y HOLDEN, L. (2001). Human Resource Management: a contemporary approach. London: Harlow, Ed. Financial Times/Prentice Hall

BEAUVALLET, G. y HOUY T. (2007). "Continous improvement of research on lean management", Working Paper in Economics and Social Sciences, ESS07-18, Telecom Paris, Paris

BENDERS, J., HUIJGEN, F., PEKRUHL, U. y O'KELLY, K. (1999). Useful but unused - groupwork in Europe, Dublin: European Foundation for the Improvement of Living andWorking Conditions

BENNETT, B. (2003). "Job Rotation. Its role in promoting learning in organizations". Development and Learning in Organizations, 17, pp. 7-9

BERGGREN, CH. (1993). The Volvo experience. Alternative to lean production in swedish auto industry. Basingstoke: Palgrave Mcmillan

BIRDI, K., CLEGG, C., PATTERSON, M., ROBINSON, A., STRIDE, C., WALL, T. y WOOD, S. (2008). "The impact of human resource and operational management practices on company productivity: a longitudinal study". Personnel Psychology, 61(3), pp. 467501

BLASI, J.R., FREEMAN, R.B., MACKIN, C. y KRUSE, D.L. /2010). "Creating a Bigger Pie?- The effects of ownership, profit sharing, and stock options on workplace perfomance". En D.L. Kruse, D.L:, Freeman, R.B y Blasi, J.R. (eds.). Shared capitalism at work: employee ownership, Profit and gain sharing, and broad-based stock options. National Bureau of Economic Research. University of Chicago Press, pp. $139-165$

BONAVIA, T. y MARÍN, J.A. (2010). "Producción ajustada y recursos humanos: resultados sobre la efectividad empresarial". Revista Europea de Dirección y Economía de la Empresa, 19 (4), pp.117-134

BOOTH, A. y FRANK J. (1999). "Earnings, productiv- 
ity, and performance-related pay", Journal of Labor Economics, 17 (3), pp. 447-463

BOWIE-McCOY, S. W., WENDT, A. C., y CHOPE, R. (1993). "Gainsharing in public accounting: Working smarter and harder". Industrial Relations, 32 (3), pp.432-445

BOYER, R. y FREYSSENET, M. (2000): Les modeles productifs. La Découverte, Collection Repéres, Paris

BRESNAHAN, T., BRYNOLFSSON, E. y HITT, L. (2002). "Information Technology, Workplace Organization, and the Demand for Skilled Labor: Firm-Level Evidence". Quarterly Journal of Economics, 117 (1) , pp. $339-378$

BULLINGTON, K.E. (2003). "5S for suppliers", Quality Progress, 36 (1), pp. 56-61,

CABLE, J. y WILSON, N. (1990). "Profit-sharing and productivity. Some further evidence", Economic Journal, 100 (401), pp. 550-555

CAHUC, P. y DORMONT, B. (1997). "Profit sharing: does it increase productivityand employment". A theoretical model and empirical evidence on french micro data". Labour Economics, 4, pp. 293-319,

CAPELLI, P. y NEUMARK, D. (2001). "Do high perfomance work practices improves establishment level outcomes". Industrial an Labor Relations Review, 54 (4), pp. 737-775

CHAN, L.L.M., SHAFER, M.A., y SNAPE, E.(2004). "In search of sustained competitive advantage: The impact of organizational culture, competitive strategy and human resource management practices on firm performance". International Journal of Human Resource Management, 15 (1), pp.17-35.

CHAPMAN, C.D. (2006). "Clean house with lean 5.". Qualitry Progress, 38 (6), pp. 27-32

CHE, Y.K. y YOO, S. W. ( 2001). "Optimal Incentives for Teams". American Economic Review, 91(3), pp. $525-541$

CHUNG, K. A. y ROSS, M. F. (1977). "Differences in motivational properties between job enlargement and job enrichment". The Academy of Management Review, 2 (1), pp. 113-122

CORDERY, J. L. (1989). "Multi-skilling: a discussion of proposed benefits of new approaches to labour flex- ibility within enterprises". Personnel Review, 18 (3), pp. $13-22$

CORIAT, B. (1992). El taller y el cronómetro. Ensayo sobre el taylorismo, fordismo y la producción en masa, Madrid: Siglo XXI

COSGEL, M.N.y MICELI, T.J. (1998). "On job rotation, Economic". Working Papers, 1998/02, University of Connecticut, USA

COSGEL, M.N.y MICELI, T.J. (1999). "Job rotation: Cost benefits, and stylized facts". Journal of Institucional Economics, 155 (2), pp. 301-320

COX, A. (2005). "The outcomes of variable pay systems: tales of multiple costs and unforeseen consequences". International Journal of Human Resource Management 16 (8), pp.1475-1497

CRUZ, K.S. y PIL, F.K. (2011). "Team design and stress: A multilevel analysis", Human Relations, 65 (9), pp.1265-1289

CUA, K.O., McKONE; K.E: y SCHROEDER, R.G. (2001). "Relatiohship between implementation of TQM, JIT, TPM and Manufacturing Perfomance", Journal of Operation and Management, 19 (6), pp.675-694

CUATRECASAS, L. (2000). TPM. Hacia la competitividad a través de la eficiencia de los equipos de producción, Gestión 2000, Barcelona

CUATRECASAS, L., OLIVELLA, J. (2006). "Aproximación a los principios del trabajo para lean", X Congreso de Ingeniería de Organización. Valencia, 7 y 8 de septiembre de 2006

CULLY, M., WOODLAND S. O'RELLY, A. y DIX, G. (1999): Britain at Work: As depicted by the 1998 Workplace Employee Relation Survey, Routledge, Londres

D'ART, D. y TURNER, T. (2006). "Profit sharing, firm perfomance and union influence in selected European countires", Personnel Review, 33 (3), pp. 333350

D'AVENI, R.A. (1994). Hypercompetition: Managing the dynamics of manoeuvring, Free Press, New York.

DALE, B.G. y BOADEN, R.J. (1994). "The use of teams in quality improvement”. En B.G. Dale (ed.): Manag- 
ing Quality, Prentice Hall, Nueva York, pp.514-530

DANFORD, A., RICHARDSON, M., STEWART, P., TAILBY, S. y UPCHURCH M. (2008). 'Partnership, High Performance Work Systems and Quality of Working Life'. New Technology, Work and Employment 23(3), pp. 51-166

DATTA, D.K., GUTHRIE, J.P. y WRIGTH, P.M. (2005). "HR and labor productivity: Does industry matter?". Academy of Management, 48 (1), pp. 135-145

DE VARO, J. (2006). "Teams, autonomy, and the financial performance of firms: New evidence from panel data". Industrial Relations, 45 (2), pp. 217-269

DELANEY, J.T. y HUSELID, M.A. (1996). "The impact of human resource management practices on perception of organizational perfomance". Academy of Management Journal, 39 (4), pp. 949-969

DELARUE, A., VAN HOOTEGEM, G., PROCTER, S. y BURRIDGE, M. (2008). "Teamworking and organiztional perfomance: A review of survey-based research". International Journal of Management Review, 10 (2), pp. 127-148

DELBRIDGE, R., LOWE, J. y OLIVER, N. (2000). "Shopflorr responsabilities under teamworking". Human Relations, 23 (11), pp. 1459-1479

DOUCOULIAGOS, C. (1995). "Worker participation and productivity in labor managed and participatory capital firms. A meta-analysis". Industrial and Labor Relations Review, 49 (1), pp. 58-77

EPOC (1997). New forms of work organization. Can Europe realise it potential?. European Foundation for The Improving of Living and Working Conditions, Luxemburg: Office of Official Publications in The European Communities.

ERIKSSON, T. (2003). "The effects of new work practices: Evidence from employer-employee data". En $\mathrm{T}$.

Kato Kato and Jeffrey Pliskin (eds.): Advances in the Economic Analysis of Participatory and Labor Managed

Advances in the Economic Analysis of Participatory \&amp; Labor-Managed Firms, Volume 7), Emeraild Group
Publishing Limited Oxfrod, UK, pp. 3-30

ERIKSSON, T. y ORTEGA, J. J. (2006). "The adoption of job rotation: Testing the theories". Industrial and Labor Relations Review, 59 (4), pp. 652-656

EWING, BT. (1996). "Wages and perfomance based pay evidence from the NLSY". Economic Letters, 51 (2), pp. 241-246

FARIÑAS, J.C. y FERNÁNDEZ DE GUEVARA, J. (dir.) (2014). La empresa española ante la crisis del modelo productivo: Productividad, competitividad en innovación, Fundación BBVA, Bilbao

FERNÁNDEZ RÍOS, M., RICO, R., MARTíN, R.S., and De la Corte, L. (2005). "Spanish firms flexibility". Psicothema, 17 ( 4), pp. 620-626

FINA, S. L. (2001). El reto del empleo. Mc Graw Hill, Madrid

FLOOD, P., GUTHRIE, J., LIU, W. \& MACa CURTAIN, S. (2005). High Performance Work Systems in Ireland: the Economic Case. Dublin: NCPP

FLOOD, P., G GUTHRIE, J., LIU, W., ARMSTRONG, C., MAC CURTAIN, S., MKAMWA, T. y O'REGAN, C. (2008). New Models of High Performance Work Systems: The Business Case for Strategic HRM, Partnership and Diversity and Equality Systems. Equality Authority and National Centre for Partnership \& Performance, Dublin

FORTUNY, J, y CUATRECASAS, L. (2003). "La necesidad del kaizen en la ISO 9000:2000", V Congreso de Ingeniería de Organización, Valladolid-Burgos, 4-5 septiembre

FRENKEL, S. y KURUVILLA, S. (2002). "Logic of action, globalization, and employee relations change in China, India, Malasia y Filipinas". Industrial \& Labor Relations Review, 55 (3), pp. 387-412

FRIEDRICH, A. KABST, R. WEBER, E., RODEHUTH, M. (1998). "Functional flexibility merely reacting or acting strategically?". Employee Relations, 20 (5), pp. $504-523$

GARCÍA OLAVERRI, C. y HUERTA ARRIBAS, E. (1999). "La innovación en la empresa española. Extensión de los nuevos sistemas de organización del trabajo". Economia Industrial, 329, 43-56. 
GARCÍA OLAVERRI, C. y HUERTA ARRIBAS, E. (2012). "Why do some companies adopt advanced management systems. The Spanish case". Management Research: The Journal of Iberoamerican Academy of Management, 10 (2), pp. 99-124

GARCIA LORENZO, A. y PRADO, J.C. (2002). La participación del personal en la mejora continua en las empresas, Madrid: Asociación Española para la Calidad

GIELEN, A., KERHOFS, M., VAN OURS, J. (2006). Performance Related Pay and Labor Productivity. IZA Discussion Paper, Nº 2211

GIELEN, A., KERHOFS, M., VAN OURS, J. (2010). "How performance related pay affects productivity and employment". Journal of Population Economics, 23(1): pp. 291-301

GITTLEMAN, M., HORRIGAN, M., JOYCE, M. (1998). "Flexible workplace practices: evidence from a nationally representative survey". Industrial and Labor Relations Review, 52 (1), pp. 99-115

GONG, Z. y HU, S. (2008). "An economic evaluation model of product mix flexibility". The International Journal of Management Science, 36 (5). 852-864

GOUDSWAARD, A., y DE NANTEUIL, M. (2000). Flexibility and working conditions. A qualitative and comparative study in seven EU Member States, Dublin: European Foundation for the Improvement of Living and Working Conditions

GOUDSWAARD, A., OEIJ, P., BRUGMAN, T. y DE JONG, T. (2005). Good practice guide to internal flexibility policies in companies, Dublin: European Foundation for the Improvement of Living and Working Conditions

GUTHRIE, J.P. (2001). "High involvement work practices: turnover and productivity: evidence from New Zealand". Academy of Management Journal, 44, 1, pp.180-190

GUTHRIE, J. P. (2008). High Perfomance Work Systems, workforce productivity, and innovation: A comparison in of MNCs and Indigenous Firms, Link Working Paper series, The Learning Innovation and Knowledge Research Centre, DCU Business School, Dublin City University

GUTIÉRREZ BRONCANO, S., RUBIO ANDRÉS, M. y MONTOYA MONSALVE (2011). "La organización flexible y su influencia en la implantación de prácticas de alto rendimiento: un estudio empírico". Cuad- ernos de Estudios Empresariales, 21, pp. 67-95

GRÚTTER, A. W.; FIELD, J. M.; FAULL, N. H. B. (2002). "Work team performance over time: three case studies of South African manufacturers". Journal of Operations Management, 20 (5), pp. 641-657

HAMILTON, B.H., NICKERSON, J.A. y OWAN, H. (2003). "Team incentives and worker heterogeneity. An empirical analysis of the impact of teams and productivity and participation". Journal of Political Economy, 111 (3), pp. 465-497

HANDEL, M.J. y GITTLEMAN, M. (2004). "Is there a wage payoff to innovative work practices". Industrial Relations, 43 (1), pp. 67-97

HARTENIAN, L.S. (2003). "Team member acquisition of team knowledge, skills and abilities". Team Perfomance Management. An International Journal, 9 (1/2), pp. 23-30

HOWARD, L.W. y DOUGHERT, T.W (2004). "Alternative reward strategies and employee reactions". Comepensation and Benefits Review, 36 (1), pp. 4151

HSU, I.C., LIN, C.Y.Y., LAWLER, J.J. y WY, S.H. (2007). "Toward a model of organizational human capital development: Preliminary evidence from Taiwan". Asia Pacific Business Review, 13 (2), pp. 251-275

HUANG, H.J. (1999): "Job rotation from the employees point of view". Human resource management, 7 (1), pp. $75-85$

HUERTA ARRIBAS, E., BAYO MORIONES, J.A., GARCÍA OLAVERRI, C. y MERINO, J. (2003). Los desafíos de la competitividad. La innovación organizativa y tecnológica en la empresa española. Bilbao: Fundación BBVA

HUSELID, M. (1995). "The impact of human resource management practices on turnover, productivity and corporate financial perfomance". Academy of Management Journal, 38 (3), pp. 635-672

ICHINIOWSKI, C., SHAW, K. y PRENNUSHI, G. (1997). "The effects of human resource management on productivity: a study of steel fihishing line". American Economic Revew, 87 (3), pp. 291-313

ILO (2003). Best practices in work-flexibility schemes and their impact on the quality of working life in the chemical industrie, Report for discussion at the Tripartite Meeting on Best Practices in Work-flexibility Schemes and theri impact on Quality Working Life in 
Chemical Industries, Geneva: International Labour Office

IMAI, M. (1986): Kaizen: the key to Japan's Competitive Sucess, New York: Random House

INSTITUTE OF MANAGEMENT ACCOUNTS (1994). Managing cross functional teams, Leadership strategies and ethics, Institute of Management Accountant

JACKSON, P.R. y MARTIN, R. (1996). "The impact of Just in Time on job content employee attitudes and well-being. A longitudinal study". Ergonomics, 19 (1), pp. 1-16.

JONES, D.C., KALMI, P., KAUHANEN, A. (2010). "Team, perfomance related pay, profit-sharing and productive efficiency. Evidence from a food-processing plant". Industrial and Labor Relations Review, 63 (4), pp. 606-626

JORGERSEN, F., BOER, H. and GERTSEN, F. (2004). "Development of a team-based framework for conducting self-assessment of continuous improvement". Journal of Manufacturing Technology Management, 15 (4), pp. 343-349

JANAana, F. y PETR., P. (2013). "Profit sharing - A tool for imptoving productivity, profitability and competitiveness of firms?". Journal of competitiveness, 5 (4), pp. 3-25

JASSAWALLA, R. y SASHITTAL, H.C. (1999). "Buliding collaborative cross.functional teams". Academy of Management Executive, 13 (3), pp. 50-63

JEREZ GÓMEZ, P., CÉSPEDES LORENTE, J.J. y VALLE CABRERA, R. (2004). "Training practices and organizacional learning capability. Relationship and implications". Journal of European Industrial Training, 28 (2/3/4), pp. 234-256

KALLEBERG, A. (2001): "Organizing flexibility: the flexible firm in a New Country". British Journal of Industrial Relations, 39 (4), pp. 479-504.

KALMI, P., PENDLETON, A. y POUTSMA, E. (2005): "Financial participation and perfomance in Europe". Human Resource Management Journal, 15 (4), pp. 54-67

KARLSSON, CH. y ÁHLSTRÖN, P. (1995): "Change processes towards lean production: the role of the remuneration systen". International Journal of Operation \& Production Management, 15 (11), pp. 80-89
KARLSSON, CH. y ÁHLSTRÖN, P. (1996), "Change processes towards lean production. The role of the management accounting system". International Journal of Operations \& Production Management , 16 (11), pp. 42-56

KARUPPAN, C.M. (2004). "Strategies to foster labor flexibility". International Journal of Productivity and Performance Management, 53 (7), pp. 532-54

KATO, T. y MORISHIMA, M. (2003). "The nature, scope and effects of profit-sharing in Japan: evidence from new survey data". International Journal of Human Resource Management, 14 (6), pp. 942-955.

KAUHANEN, A. (2009). "The incidence of High-Perfomance Work System: evidence from a nationally representative employee survey". Economic and Industrial Democracy, 30 (3), pp. 454-480

KAYMAZ, K. (2010). "The effects of job rotation practices on motivation: A research on managers in the automotive organizations". Business and Economics Research Journal, 1 (3), pp. 69-85

KERSLEY, B., ALPINI, C., FORTH, J., BRYSON, A., BEWLEY, H., DIX, G. y OXENBRIDGE, S. (2006). Inside the workplace: finding from the 2004 workplace employment relation survey, London: Routledge

KITSON, M., y WILKINSON, F. (1999)."Training, Human Resources Management methods and the national minimum wage". En A. Cosh \& A. Hughes (Eds.): British Enterprise in Transition Cambridge, Cambridge: Centre for Business Research

KOIKE, K. (1988). Understanding industrial relations in modern Japan, Basingstoke: Macmillan

KOIKE, K. (1991). Shigoto no Keizaigaku. Tokio: ToyoKeizai Shinpo

KOZLOWSKI, S.W. y Bell, B.S. (2003). "Work groups and teams in organiztion". En W. C. Borman, D. R. Ilgen, \& R. J. Klimoski (Eds.), Handbook of psychology: industrial and organizational psychology, Hoboken $\mathrm{NJ}$ : Wysley, pp. 333-375

KRUSE, D.L: (1992). "Profit sharing and labor productivity: microeconomic evidence from the United States". Economic Journal, 102, pp. 24-36

KRUSE, D.L. (1993a). Does profit sharing affect productivity?. NBER Working Paper, Nº 4542, Noven- 
ber

KRUSE, D.L. (1993b). Profit sharing. Does it make a difference?, Kalamazoo, MI: W.E. Upjohn Institute

KRUSE, D.L. (1996). "Why do firms profit-sharing and employee ownerships plant?". British Journal of Industrial Relations, 34 (4), pp. 515-538

KYZLINKOVÁ, R.; DOKULILOVÁ, L.; KROUPA, A. (2007). Teamwork and high performance work organization, Dublin: European Foundation for the Improvement of Living and Working Conditions

LAWLER, E., MOHRMAN, S. y LEDFORD, G. (1992). Employee involvement and Total Quality Management: Practices and results in Fortune 1000 companies, San Francisco: Jossey-Bass

LAWLER, E.E., MOHRMAN, S.A. y LEDFORD, G.E. (1995). Creating High Perfomance Organizations: Practices and results of employee involvement and Total Quality Management in Fortune 1000 companies, San Francisco: Jessey-Bass

LAWLER, E.E. III (2000). Rewarding excellence: Pay strategies for the new economy, San Francisco: Jossey Bass

LAWLER, E.E., MOHRMAN, S.A. y BENSON, G. (2001). Organizing for High Perfomance: rmployee involvement, TQM, reengineering and knowldege in the Fortune 1000, San Francisco: Jossey-Bass Wiley

LAWLER, E.E., MOHRMAN, S.A. (2003). Pay practices in Fortune 1000 comporations. Los Angeles: Center for Effective Organizations, University of Southern California

LAZEAR, E.P. (2000). "Perfomance pay and productivity", American Economic Revew, 90 (5), pp. 13461361

LAZEAR, E.P.. (2004). "Output-based pay: incentives, retention or sorting”. En:S.W. Polachek (ed.), Research in Labor Economics, Stanford: JAI Press, pp. 1-25

LAZEAR, EP; SHAW, KL (2007). "Personnel economics: The economist's view of human resources". Journal of Economic Perspectives, 21 (4), 91-114.

LEDFORD, G.E. (1995). "Paying for the skills, knowledge, and competencies of knowledge workers",
Compensation \& Benefits Review, 27 (4), pp. 55-62

LEPAK, D.P., TAKEGUCHIi, R., SNELL, S.A. (2003). Employment flexibility and firm performance: examining the interaction effects of employment mode, environmental dynamism, and technological intensity. Journal of Management, 29(5), pp. $681-703$

LEWCHUCK, W. y ROBERTSON, D. (1997). "Production without empowerment. Work reorganization from perspective of motor vehicle worker". Capital and Class, 21 (3), pp. 37-64.

LIKER, J., and HOSEUS, M. (2008). Toyota Culture, New York: McGraw Hill

LIKER, J. (2004). The Toyota Way, New York: Mc Graw Hill,

LLORENTE, F. (2007). "La flexibilidad laboral de los proveedores directos en Cataluña de los fabricantes de automóviles". Investigaciones Europeas de Dirección y Economía de la Empresa, 13 (1), pp. 149166.

LLORENTE, F. (2008). La innovació com a estratègia empresarial per a la competitivitat del sector automobilístic, Barcelona: CTESC, Generalitat de Catalunya

LLORENTE, F. (2013). "La flexibilidad laboral en la industria auxiliar del automóvil en Cataluña". Dirección y Organización, 51, pp. 65.85

LLORENTE, F. (2014). "La organización laboral y los sistemas de organización en la industria auxiliar de Cataluña". Investigaciones Europeas de Dirección y Economía de la Empresa, 20 (3), pp. 140-150

LONG, R.J. (1997). "Motives for profit sharing: A study of canadian chief executives officers". Industrial Relations, 52 (4), 712-733

LONG, R.J. y FANG, T. (2012). "Do employees profit from profit sharing?. Evidence from canadian panel data". Industrial and Labor Relations Review, 65 (4), pp. 899-927

LONG, R.J. y FANG, T. (2013). "Profit sharing and workplace productivity: Does teamwork play a role?", IZA, Discussion Paper, № 7869

LORENZ, E. y VALEYRE, A. (2005). "Organizational innovation: human resource management and labour 
market structure: a comparison of the EU-15". The journal of Industrial Relations, 47 (4), pp. 424-442

MACDUFFIE, J.P. (1995). "Human resource bundles and manufacturing perfomance: Organisational logic and flexible production systems in the world auto industry". Industrial and Labor Relations Review. 48 (2), pp. 197-221.

MAELLA, P. (2012). Retribución variable y motivación: retos y recomendaciones. IESE Ocasional Paper, 252, IESE Business School, Universidad de Navarra.

MARSCHAK, J. y RADNER, R. (1972). The theory of teams, New Haven and London: Yale University Press

MARONNAT-GEFFROY, B. (2001). "Le modèle H en question". Revue Française de Gestion, 132, janier, fevrier, pp. 44-55

MARTíNEZ, A., VELA, M.J., PÉREZ, M., DE LUIS CARNICER, y PÉREZ, M. (2010). "Externalización, flexibilidad laboral y resultados en la empresa española". Economía Industrial, 375, pp. 217-225

MARTÍNEZ-SÁNCHEZ, A., VELA-JIMÉNEZ, M.-J., PÉREZ-PÉREZ, M., y DE LUIS CARNICER, P. (2011). "The dynamics of labour flexibility: Relationships between employment type and innovativeness". Journal of Management Studies 48 (4), pp. 715-736

MCNABB, R. y WHITFIELD, K. (1998). "The impact of financial participation and employee involvement", Scottish Journal Journal of Political Economy, 45 (2), pp. 171-187

MCNABB, R. y WHITFIELD, K. (2007). "The impact of varying types of perfomance-related pay and employee participation on earning". International Journal of Human Resource Management, 18 (6), pp. 1004-1025

MELFORD, R.N. (2009). "Increasing productivity in global firms. The CEO challenge". Journal of International Management, 15 (3), pp. 262-273

MICELI, M.P.y MULVEYulvey, P.W. (2000). "Consequences of satisfaction with pay systems: two field studies". Industrial Relations, 39 (1), pp. 62-87
MICHIIE, J. y SHEEBAN, M. (2001). "Labor market flexibility, human resource management and corporate perfomance". British Journal of Management, 12 (4), pp. 287-306

MILLER, F.C., DHALIWAL, T.S., y MAGAS, L.J.(1973). "Job rotation raises productivity". Industrial Engineering, 5, pp. 24-26

MILGROM, P. y ROBERTS, J. (1995). "Complementarities and fit: strategy, structure and organizational change in manufacturing". Journal of Accounting and Economics, 19 (2-3), pp. 179-208

MITCHELL, D.B.J., LEWIN, D. y LAWLER, E.E. (1990). "Alternative pay systems, firms perfomane and productivity", In A.S. Blinder, (ed.): Paying for productivity: a look at the evidence, Washington: The Brooking Institution, pp. 15-94

MONDEN, Y. (1983). Toyota Production System - A practical approach to production management, Atlanta: Industrial Engineering and Management Press

MUELLER, F. (1992). "Designing flexible teamwork, comparing German and Japanese approaches". Employee Relations, 4 (1), 5-16

MURRAY, B. y GERHART, B. (1996). "An empirical analysis of a skilled-based pay program and plant perfomance outcomes". Academy of Management Journal, 41 (1), 68-78

NAVEEN, S. y GANESH KUMAN, N. (2012). "Quality improvements by implementing lean manufacturing principles". Advanced Materials Research, 488-489, pp. 1168 - 1173

NERGAARD, K:, DOLVIK, J: E., MARGINSON, P., ARASANZ, J. y BECHTER, B. (2009). "Engaging with variable pay: a comparative study of metal industry". European Journal of Industrial Relations, 15 (2), pp. 125-146

NIEPCE, W. y MOLLEMAN, E. (1996). "A case study. characteristic of work organization in lean production and sociotechnical systems". International Journal of Operations and Production Management, Special Issue, 16 (2), pp. 77-90

NONAKA, I. y TAKEUCHI, H. (1995). The knowledge creating company: how japanese companies create the dynamics of innovation, Oxford: Oxford University Press 
NOVELLA, J: y ALUJA, J.A. (2008). "Negociación colectiva y sistemas de remuneración variable en el sector de maquinaria en España: estudio de casos". Trabajo: Revista andaluza de relaciones laborales, 21 , pp. $200-220$

OBLÓJ, K. CUSHMAN, D.P.y KOZMINKI., A. K. (1995). Continuous Improvement Theory in high performance organizations, Albany: SUN Press

OCDE (1987). La flexibilidad en el mercado de trabajo, Madrid: MTSS

OCDE (1999). OCDE Employment Outlook June, Paris.

OECD (1995). Profit - sharing in OECD countries, En OECD Employment Outlook July, Paris, pp. 139-169

OHNO, T. (1988): Toyota Production System - Beyond Large Scale Production, Cambridge MA: Productivity Press

OLIVELLA, J., CUATRECASAS, L., y GAVILÁN, N. (2008). "Work organization practices for lean production.". Journal of Manufacturing Technology Management, 17 (7), pp. 798-811

ORIGO, F. (2009). "Flexible pay, firm performance and the role of unions. New evidence from Italy". Labour Economics, 16 (1): pp.64-78

ORIGO, F. y PAGANI, L. (2008). "Workplace flexibility and job satisfaction. Some evidence from Europa". International Journal of Manpower, 29 (1), pp. 42-48

ORTEGA, J. (2001). "Job rotation as a learning mechanism”. Management Science, 47 (10), October, pp. $1361-1370$

ORTIZ, L. (1999). Convergencia o permanencia de los sistemas de relaciones laborales: reacción sindical a la introducción del trabajo en equipo en la industria del automóvil, Instituto Juan March de Estudios e Investigaciones, Centro de Estudios Avanzados en Ciencias Sociales, Madrid.

OSTERMAN, P. (1994). "How common on workplace transformation and who adopts it?". Industrial and Labor Relatons Review, 47 (2), pp. 173-178

OSTERMAN, P. (2000). "Work reorganization in an era of reestructuring: trend in diffusion and effects on employee welfare". Industrial \& Labor Relations Review, 53 (2), pp. 179-196
OSTERMAN, P. (2006). "The wage effects of High Perfomance Work Organization in manufacturing". Industrial and Labor Relations Review, 59 (2), pp. 1878-204

PARENT, D. (2002). "Perfomance pay in the United States. Its determinants and effects". En M. Brown and J.S. Heywoord (eds.):Paying for perfomance: An international comparison, Armonk, NY: M.E. Sharpe Publishers, pp. 17-51

PAENT-THIRION, A., FERNÁNDEZ MACÍAS, E., HURLEY, J. y VERMEYLEN, G. (2007). Fourth European Working Conditions Survey, Luxembourg: Office for Official Publications of the European Communities

PARKER, G. M. (2003). Cross functional teams. Working with allies, enemies and other strangers, San Francisco: Jhon Willeys and Sons

PENDLETON, A, POUTSMA, E.; VAN OMMEREN, J.; BREWSTER, Ch. (2001). Employee share ownership and profit-sharing in the European Union, Dublin: European Foundation for the Improvement of Living and Working Conditions

PÉREZ-FERNÁNDE DE VELASCO, J.A. (1999). Gestión de la calidad orientada a procesos. Madrid: Esic Editorial

PIEKKOLA, H. (2005). "Pefomance-related pay and firm perfomance in Finland", International Journal of Manpower, 26 (7/8), 619-635

PFEFFER, J.(1995). Competitive advantage through people: Unleashing the power of the workforce, Boston MA: Harvard Business School Press, Boston

PFEFFER, J. (1998). La ecuación humana. La dirección de recursos humanos clave para la excelencia empresarial. Barcelona: Gestión 2000 y Edipe

PIL, F.K. y FUJIMOTO, T. (2007). "Lean and reflexive production: the dynamic nature of production models", Internacional Journal of Production Research, 45 (6), pp. 3741-3761

PIORE, M.J. y SABEL, CH. F. (1994): The second industy divide, New York: Basic Book

POUTSMA, E., (2001). Recent trends in employee financial participation in the European Union, Dublin: European Foundation for the Improvement of Living and Working Conditions 
POUTSMA, E., HENDRICKK, J.y HUIJGEN,F.(2003). "Employee participation in Europe:in search of the participative workplace". Economic and Industrial Democracy, 24 (1), pp.45-76

PROCTER, S y MUELLER, F. (2000). 'Teamworking: strategy, structure, systems and culture'. En S. Procter and F. Mueller (eds) Teamworking. London: MacMillan Business

PUIG-BERNABEU, C. GARCÍA-SABATER, J.P., GARCÍA SABATER, J.J. y MARÍN-GARCÍA, J.A. (2010): "Gestión de reuniones en los equipos de mejora", Working Papers on Operations Management. Universidad de Valencia ,1 (2), pp. 6-12

PUVAMASVARAN, F, P., MEGAT, H., HONG, T.S., RAZALL, M.M. y MAGID, H.A. (2010). "Lean process management implementation through enhanced problem solving capabilities". Journal of Industrial Engineering and Management, 3 (3), pp. 447-493.

PRADO, J. C. (2000). El proceso de mejora continua en la empresa. Madrid: Pirámide

PRUJIT, H: (2003). "Teams between neo-taylorism and anti-taylorism", Economic and Industrial, 21 (1), pp. 71-101

RAMSAY, H., SCHOLARIS, D. y HARLEY, B. (2000). "Employee and high-performance work systems: testing inside the black box". British Journal of Industrial Relations, 38 (4), pp. 501-531

RIEDMANN, A. VAN GYES, G., ROMÁN, A., KERKHOFS, M. y BECHMANN, S. (2010). European Company Survey 2009, Overview, Dublin: European Foundation for the Improvement of Living and Working Conditions. Luxemburg: Office for Official Publications of the European Communities

ROBINSON, A.M. y WILSON, N. (2006). "Employee financial participation and productivity. An empirical reapparisal". British Journal of Industrial Relations, 44 (1), pp. 31-50

RISSEN, D., MELIN, B., SANDSJÓ, L., DOHNS, I. y LUNDLBERG, U. (2002). "Psychosphysiological stress reactions, trapezius muscle activity, and neck and shoulder among female cashiers before and after introduction job rotation". Work \& Stress, 16 (2), pp. $127-137$

SCHOUTETEN, R. y BENDERS, J. (2004). "Lean production assessed by Karasek' job demand job control model". Economic and Industrial Democracy, 25 (3), pp. 347-373'
SCOTT, A.L. (2005). "Lean manufacturing in the mill room - improve efficiencies, decrease costs and improve quality". Rubber World, 231 (6), pp. 20-21

SINGH, T.P. y CHAUHAN, G. (2013). "Significant parameters of labour flexibility contributing to lean manufacturing". Global Journal of Flexible System Management, 14 (2), pp. 93-105

SODENKAMP, D., SCHIMIDT, K., KLEINBECK, U. (2005). "Self-management of work groups through corporate values: from theory to practice. International Journal of Manpower, 26 (1), pp. 67-79

SPEAR, S. y BOWEN, H.K. (1999). "Decoding the DNA of the Toyota Production System". Harvard Business Review, 77 (5), pp. 96-106

STEVENS, M.J. y CAMPION, M.A. (1994). "The Inowledge, skill, and ability requirements for teamwork: implications for Human Resource Management". Journal of Management, 20 (2); pp. 503-530

SUNDSTROM, E., DE MEURSE, K. y FUTRELI, D. (1990). "Work teams: applications and effectiveness". American psychologist, 45 (2); pp. 120-133

SUZAKI, K. (1993). The new shop floor management: empowering people for continuous improvement, New York: Free Press

SWEINS, Ch., KALMI, P. /2008). "Pay knowledge, pay satisfaction and employee commitment: evidence from Finnish profit-sharing schemes". Human resource Management Journal, 18 (4), pp. 366-385

TANGIAN, A.S. (2007). Is flexible work precarious?. A study based on the 4th European Survey of Working Conditions, 2005, WSI-Diskussionspapier Nr.15, June

TERZIONVSKI, M. y SHOAL, A.S. (2000). "The adoption of continuous improvement and innovation strategies in Australian manufacturing firms". Technovation, 20 (10), pp. 539-550

TREMBLAY, M. y SIRE, B. (1999). "Rémunèrer les compétences plûtot que l'activité. Revue Française de Gestion, 126, Novembre-Décembre, pp. 129-139

TREY, P. (2003). Le 5S, socle de l'efficacité industrielle: mode de l'emploi, Paris: AFNOR

TSIPOURI, L., GAVROGLOU, S., ASPLUND, R. KLEINKECHTT, A.H, WICKHAM, J. ARVANITIS, S. (2005). Flexibility and competitiveness: labour mar- 
ket flexibility, innovation and organisational performance (FLEX-COM) Final report 279-287, Luxemburg: Office for Official Publications of the European Communities

TÜSELMANN, H. (1996). "Progress toward greater labour flexibility in Germany. The impact of recent reforms". Employee Relations, 18 (1), pp. 50-67

URTASUN-ALONSO, A., LARRAZA-KINTANA, M., GARCÍA-OLAVERRI, C. y HUERTA-ARRIBAS, E. (2014). "Manufacturing flexibility and advanced human resource management practices". Production Planning \& Control: The Management of Operations", 25 (4), pp. 303-317

VALEYRE, A., CANTRON, D., CSIZMADIA, P., GOLLAC, M., MAKÓ, C. (2009). Working conditions in the European Union: Worker organization, European Foundation for the improvement of living and working condition, Luxemburg: Office for Oficial Publications of the European Communities

VALVERDE, M., TREGASKIS, O. y BREWSTER, C. (2000). "Labor flexibility and firm performance". International Advances in Economic Research, 6 (4), pp. 49-661.

VALLAS, S. P. (2003). "Why Teamwork fails: obstacles to workplace change in four manufacturing plants". American Sociological Review 68 (2), pp. 223-50

VAN HET KAAR, R. y Grünell, M. (2001). Variable pay in Europe, EIROnline, http://www.eurofound.europa. eu/eiro/2001/04/study/index.html
VOUDOURIS, I. (2007). "The co-evolution of functional and numerical flexibility: do technology and networking matter?". New Technology, Work and Employment 22 (3), pp. 224-245

WADHWANI, S. y WALL, M. (1990). "The effects of profit-sharing on employee, wages, stock returns and productivity. Evidence from UK data micro-data". Economic Journal, 100 (399), pp. 1-17

WEITZMAN, M.L. y KRUSE, D.L. (1990). "Profit sharing and Productivity". En A.S Blinder (eds.): Paying for productivity. A look at evidence. Center for Economic Progress and Employment series, Washington, D.C.: Brooking Institution,

WELZ, C. Y FERNÁNDES-MACÍAS, E. (2008). "Financial Participation of Employees in the European Union: Much Ado about Nothing?". European Journal of Industrial Relations, 14 (4), pp. 479-497

WELLBOURNE, T.M. y GÓMEZ MEJÍA, L.R. (1995). Gainsharing: A critical review and a future research agenda, Chars Working Paper Series=95-10, Center of Advanced Human Resource Management, Cornell University ILR School

WOMACK, J. P., JONES, D. T. y ROOS, D. (1990). The Machine that changed the world: New York: Rawson Associates

XUE, Q. H., y XU, L. (2013). "Human Resource Flexibility and Firm Performance in China", The 19th International Conference on Industrial Engineering and Engineering Management, Springer Berlin Heidelberg, pp. 401-407 